\title{
Ankyrin-Dependent and -Independent Mechanisms Orchestrate Axonal Compartmentalization of L1 Family Members Neurofascin and L1/Neuron-Glia Cell Adhesion Molecule
}

\author{
Tatiana Boiko, Max Vakulenko, Helge Ewers, Chan Choo Yap, Caren Norden, and Bettina Winckler \\ Department of Neuroscience, University of Virginia, Charlottesville, Virginia 22908
}

\begin{abstract}
Axonal initial segments (IS) and nodes of Ranvier are functionally important membrane subdomains in which the clustering of electrogenic channels enables action potential initiation and propagation. In addition, the initial segment contributes to neuronal polarity by serving as a diffusion barrier. To study the mechanisms of axonal compartmentalization, we focused on two L1 family of cell adhesion molecules (L1-CAMs) [L1/neuron- glia cell adhesion molecule (L1/NgCAM) and neurofascin (NF)] and two neuronal ankyrins (ankB and ankG). $\mathrm{NF}$ and ankG accumulate specifically at the initial segment, whereas L1/NgCAM and ankB are expressed along the entire lengths of axons. We find that L1/NgCAM and NF show distinct modes of steady-state accumulation during axon outgrowth in cultured hippocampal neurons. Despite their different steady-state localizations, both L1/NgCAM and NF show slow diffusion and low detergent extractability specifically in the initial segment but fast diffusion and high detergent extractability in the distal axon. We propose that L1-CAMs do not strongly bind ankB in the distal axon because of spatial regulation of ankyrin affinity by phosphorylation. NF, conversely, is initially enriched in an ankyrin-independent manner in the axon generally and accumulates progressively in the initial segment attributable to preferential binding to ankG. Our results suggest that $\mathrm{NF}$ and $\mathrm{L1} / \mathrm{NgCAM}$ accumulate in the axon by an ankyrinindependent pathway, but retention at the IS requires ankyrin binding.
\end{abstract}

Key words: initial segment; L1 CAMs; retention vs targeting; ankyrin; plasma membrane (axolemma); trafficking

\section{Introduction}

Compartmentalization of the neuronal plasma membrane is crucial for proper neuronal function. Distinct functional domains, such as axons and dendrites, differ from each other morphologically, functionally, and by molecular composition. The specific functions of axons and dendrites are performed by specialized sets of membrane molecules that first have to be properly localized to their sites of function. The axonal initial segment (IS) is a highly specialized subdomain of the axon in which membrane proteins [such as voltage-gated sodium channels (VGNCs), po-

\footnotetext{
Received 0ct. 2, 2006; revised Nov. 26, 2006; accepted Dec. 4, 2006.

This work was supported by a Revson Foundation Fellowship (to T.B.), National Institutes of Health Grant 1R01NS 045969-01, a Whitehall Foundation Fellowship, and a Scientist Development grant from the American Heart Association (all to B.W.). We thank the following people for generously sharing reagents: Vance Lemmon (University of Miami, Miami, FL), Vann Bennett (Duke University, Durham, NC), Matthew Rasband (University of Connecticut Health Center, Farmington, (T), Peter Brophy (Edinburgh University, Edinburgh, UK), Dan Felsenfeld [Mt. Sina School of Medicine (MSSM), New York, NY], Ira Mellman (Yale University, New Haven, (T), and Michele Solimena (University of Dresden, Dresden, Germany). We thank Dan Felsenfeld (MSSM) for invaluable hands-on advice on analyzing bead trajectories. We thank David Colman, Greg Phillips, and Deanna Benson at the Fishberg Department of Neuroscience, MSSM for advice and support (for T.B.), Dan Felsenfeld (MSSM) and Frank Solomon (Massachusetts Institute of Technology, (ambridge, MA) for helpful comments on this manuscript, Dolora Wisco for expert technical assistance, and Scott Henderson (Virginia Commonwealth University, Richmond, VA) for confocal imaging support.

Correspondence should be addressed to Bettina Winckler, Department of Neuroscience, University of Virginia, 409 Lane Road, MR4-6112, Charlottesville, VA 22908. E-mail: bwinckler@virginia.edu.

DOI:10.1523/JNEUROSCI.4302-06.2007

Copyright $\odot 2007$ Society for Neuroscience $\quad$ 0270-6474/07/270590-14\$15.00/0
}

tassium channels, and cell adhesion molecules] as well as cytoskeletal components (such as specialized ankyrins and spectrins) accumulate at high density (Kordeli et al., 1995; Berghs et al., 2000; Jenkins and Bennett, 2001; Pan et al., 2006) (for review, see Salzer, 2003; Hedstrom and Rasband, 2006). As a result, the IS is a site of action potential initiation, in which neuronal firing is regulated (Coombs et al., 1957; Frankenhaeuser and Huxley, 1964; Khaliq and Raman, 2006; Palmer and Stuart, 2006; Van Wart and Matthews, 2006). Additionally, cell adhesion molecules (CAMs) clustered at the IS can serve to guide synaptogenesis during development (Ango et al., 2004). Third, using cultured neurons, the IS was shown to act as a diffusion barrier that prevents intermixing of axonal and somatodendritic membrane proteins (Winckler et al., 1999; Nakada et al., 2003).

The L1 family of CAMs consists of L1 [named neuron-glia cell adhesion molecule (NgCAM) in chick], neurofascin (NF), neuron-glia related cell adhesion molecule (NrCAM), and CHL1 (for close homolog of L1) (Hortsch, 2000). The conserved cytoplasmic tail binds ankyrin (Hortsch et al., 1998; Zhang et al., 1998) among other proteins (Kamiguchi et al., 1998; Dickson et al., 2002; Kizhatil et al., 2002; Cheng et al., 2005). Despite the high homology, L1 family members show distinct steady-state localizations in vivo (Scotland et al., 1998): L1 is found all along axons, whereas NF and NrCAM (Custer et al., 2003) are enriched at the IS. L1 family members take part in many important processes 
(such as axon outgrowth, pathfinding, fasciculation, synapse formation and function, and myelination), at least some of which require ankyrin binding (Volkmer et al., 1996; Haspel and Grumet, 2003; Kamiguchi, 2003; Sherman et al., 2005). Mutations in the neuronal cell adhesion molecule L1 are responsible for an $\mathrm{X}$-linked recessive neurological disorder [CRASH (acronym for corpus callosum agenesis, retardation, aphasia, spastic paraplegia, hydrocephalus)] that presents with varying penetrance of hydrocephalus, mental retardation, and hypoplasia/absence of the corticospinal tract and the corpus callosum (Fransen et al., 1995). Several of the $\mathrm{L} 1$ alleles associated with CRASH map to the cytoplasmic tail, one to the ankyrin binding site itself.

Ankyrins are also part of a gene family consisting of ankB, ankG, and ankR (Bennett and Lambert, 1999; Bennett and Chen, 2001). Isoforms of both ankB and ankG are expressed in neurons: ankG is enriched in the IS (Kordeli et al., 1995), whereas ankB is found all along axons (Scotland et al., 1998; Zhang and Bennett, 1998). AnkB knock-out animals die by postnatal day 21 and show abnormalities in the brain, as well as in other organs such as the heart (Scotland et al., 1998). In cardiac myocytes, Bennett's group demonstrated elegantly that ankB and ankG perform noninterchangeable functions (Mohler et al., 2002, 2005).

In this work, we compare the localization of two related CAMs, L1/NgCAM and NF, and two ankyrins, ankB and ankG, during axon outgrowth in cultured neurons. The colocalization of NF and ankG at the IS in vivo (Zhou et al., 1998; Jenkins and Bennett, 2001; Boiko et al., 2003) and in cultured neurons (Xu and Shrager, 2005; present study) suggests that the localization of $\mathrm{NF}$ is determined by its binding to ankG. Consistent with this notion, ankG deficiency leads to loss of IS localization of NF, voltage-gated sodium channels, and potassium KCNQ channels in an ankG knock-out mouse (Zhou et al., 1998; Jenkins and Bennett, 2001; Pan et al., 2006). Binding to ankyrin might, therefore, be required during secretory trafficking to the plasma membrane and/or for tethering of ankyrin-binding proteins after arrival at the cell surface (Tuvia et al., 1999; Garrido et al., 2001, 2003; Kizhatil and Bennett, 2004; Sherman et al., 2005; Xu and Shrager, 2005). Given the long time course of knock-out or knockdown experiments, it was not possible to clearly distinguish between a secretory targeting role and a post-insertion retention role for ankG. We therefore use two experimental strategies that acutely assay the behavior of the surface pool of L1 family members, i.e., retention: diffusion measurements and detergent extractions of live cells (Winckler et al., 1999). Our results suggest that NF and NgCAM accumulate in the axon by an ankyrinindependent pathway, but retention at the IS requires ankyrin binding. Compartmentalization of the axonal plasma membrane therefore occurs by multiple steps.

\section{Materials and Methods}

Antibodies

Original particle tracking experiments were done using an anti-NF186 rabbit antibody (generous gift from Dr. Peter Brophy, Edinburgh University, Edinburgh, UK). We made anti-NF186 antibodies in rabbits (named "rabbit 4753") and in chicken (chNY926) (immunized by Covance Research Products, Berkeley, CA) using a mixture of three peptides from the extracellular mucin-rich domain of NF186 conjugated to KLH (Davis et al., 1996). Rabbit anti-ankG antibody was made against the $\mathrm{KLH}$-coupled peptide representing the unique last $11 \mathrm{C}$-terminus amino acids of AnkG270 (Johnson et al., 2006). ASCS4 anti-L1 and 8D9 anti$\mathrm{NgCAM}$ hybridomas were obtained from the National Institutes of Health Hybridoma Bank. The hybridomas were grown according to the instructions of the manufacturer. Tissue culture supernatants were collected and concentrated over a T-gel column (Pierce, Rockford, IL).
Mouse anti-ankB and mouse anti-ankG were from Santa Cruz Biotechnology (Santa Cruz, CA), chicken anti-microtubule-associated protein-2 (MAP2) was from Covance Research Products, anti-tubulin (mouse anti- $\alpha$-tubulin) was a generous gift from Dr. Gianni Piperno (Mount Sinai School of Medicine, New York, NY), 32 and anti-hemagglutinin (HA) (mouse) was from Covance Research Products. Rabbit anti-L1 was a generous gift from Dr. Vance Lemmon (University of Miami, Miami, FL), anti-myc 9E10 was from Upstate (Chicago, IL). Anti-phosphoFIGQY was a generous gift from Dr. Vann Bennett (Duke University, Durham, NC). The anti-phospho-FIGQY antibody was highly purified by multiple cross-absorptions to remove antibodies binding the nonphospho-FIGQY forms (for details, see Jenkins et al., 2001). We thank Dr. Matt Rasband (University of Connecticut Health Center, Farmington, CT) for generously providing an unpublished monoclonal antibody against a non-mucin-rich domain epitope of neurofascin. The antibody against $\beta \mathrm{IV}$-spectrin was generously provided by Dr. Michele Solimena (University of Dresden, Dresden, Germany).

\section{Plasmids}

Plasmids were generous gifts, as follows: ankB-GFP, ankG-GFP, and HA-NF from Dr. Vann Bennett; NgCAM from Dr. Peter Sonderegger (University of Zurich, Zurich, Switzerland); and myc-L1 and mutants from Dr. Dan Felsenfeld (Mt. Sinai School of Medicine, New York, NY).

\section{Cell culture}

Primary cultures of hippocampal neurons were grown as described previously (Wisco et al., 2003). Embryonic day 18 rat hippocampi were dissected, dissociated by trituration after trypsin digestion, and plated on nitric-acid washed, poly-lysine-coated coverslips or cell culture dishes in Minimal Essential Medium (MEM) (Invitrogen, Carlsbad, CA) with $10 \%(\mathrm{v} / \mathrm{v})$ heat inactivated horse serum. After four hours, the cells were transferred into serum-free medium [MEM, $2 \mathrm{mM}$ L-glutamine, and $0.7 \%(\mathrm{w} / \mathrm{v})$ glucose] supplemented with B27 (Invitrogen) and cultured for 4-8 d. Depending on the experiment, they were plated on gridded coverslips (Bellco Glass, Vineland, NJ) for single-particle tracking and on round Bellco Glass coverslips in $6 \mathrm{~cm}$ dishes for extractions and immunocytochemistry.

\section{Ankyrin recruitment assay}

Human embryonic kidney HEK 293 cells were cultured in 10\% fetal bovine serum DMEM. A day before transfection, they were plated into 12 -well dishes at the density of $3 \times 10^{5}$ cells per coverslip. They were cotransfected with ankyrin [ankyrin B-green fluorescent protein (GFP) or ankyrin G-GFP] and neurofascin-HA constructs in serum-free medium using Lipofectamine 2000 according to the protocol of the manufacturer. Because the ability to observe recruitment depends on both the affinity of the NF-ankyrin interaction and the amounts and ratios of the two binding partners, we systematically varied the ratio of NF and ankyrin plasmids over a 10-fold range. Because both ankyrin isoforms were tagged with GFP, we could directly compare the abundance of the expressed ankyrins in different experiments and normalize across experiments. The amount of DNA for ankyrin B was adjusted to obtain comparable expression with that of ankyrin G. Thus, $0.7 \mu \mathrm{g}$ of HA-neurofascin was cotransfected with $0.03 \mu \mathrm{g}$ up to $0.3 \mu \mathrm{g}$ of ankyrin G-GFP or ankyrin B-GFP. Empty vector without insert was included to give a final DNA amount of $1 \mu \mathrm{g}$. Soluble ank-GFP showed characteristic cytosolic staining, leaving the nuclear region dark. The criterion for ankyrin recruitment was accumulation of GFP signal at the surface of the cell sufficient to conceal a nuclear outline.

\section{Adenoviral infection}

Hippocampal cultures on glass coverslips were moved to 12 -well plates in $500 \mu \mathrm{l}$ of conditioned medium in the presence of $1 \mathrm{~mm}$ kynurenic acid, and $1-5 \mu$ l of purified recombinant adenovirus was added per well. After $4 \mathrm{~h}, 500 \mu \mathrm{l}$ of conditioned medium was added, and cells were incubated for 24-26 h. Delta FIGQY NgCAM was kindly provided by Eric Anderson (Ira Mellman Laboratory, Yale University, New Haven, CT).

Neurons were transfected with Lipofectamine 2000 as described previously (Wisco et al. 2003). 


\section{Detergent extractions}

Neurons were extracted for $8 \mathrm{~min}$ in $800 \mathrm{ml}$ of cytoskeletal buffer $(2 \mathrm{~mm}$ $\mathrm{MgCl}_{2}, 10 \mathrm{~mm}$ EGTA, and $60 \mathrm{~mm}$ PIPES, pH 7.0) containing $0.5 \%(\mathrm{v} / \mathrm{v})$ Triton X-100 (Bio-Rad, Hercules, CA) at $37^{\circ} \mathrm{C}$ and afterward immediately fixed in $2 \%$ formaldehyde/ $3 \%$ sucrose in PBS.

\section{Immunocytochemistry}

Neurons were fixed for $20 \mathrm{~min}$ at room temperature in $2 \%$ formaldehyde $/ 2 \%$ sucrose in PBS and then washed in PBS and PBS/20 mm glycine. For surface staining, cells were blocked with 1\% BSA/PBS $6 \%$ normal donkey serum for $45 \mathrm{~min}$. For intracellular epitopes, cells were permeabilized in blocking solution containing $0.2 \%$ Triton X-100 for $20 \mathrm{~min}$ at room temperature. Primary antibody was added for 60-90 min diluted in blocking solution. The primary and secondary antibody solutions were spun for $10 \mathrm{~min}$ in the cold. Secondary was diluted in block without Triton X-100 and incubated for $40 \mathrm{~min}$, followed by washes in PBS. Coverslips were mounted in Vectashield (Vector Laboratories, Burlingame, CA). To control for binding specificity, primary antibodies were incubated with molar excess of antigenic peptides for $30 \mathrm{~min}$ at room temperature in parallel with control incubations with nonspecific peptide or protein.

HEK 293 cells were fixed with $2 \%$ paraformaldehyde for $20 \mathrm{~min}$ and then incubated in 1\% BSA, $4 \%$ donkey serum PBS blocking buffer for 10 min. Anti-HA antibody was incubated 1:500 for $1 \mathrm{~h}$. Cells were washed three times with PBS and secondary antibody was applied for $45 \mathrm{~min}$.

\section{Absence versus nondetectability}

We identify a stage of axonogenesis characterized by the absence of detectable ankG staining at the IS. Because the percentage of neurons showing nondetectable ankG staining in young cultures depends on culture conditions (such as density), this early broad NF pattern cannot be observed in all cultures. In fact, in dense cultures, ankG-negative neurons are virtually absent, even early on, and the ankG/NF-positive IS develops rapidly. In motor neuron cultures, detectable accumulation of VGNCs at the IS also occurred very rapidly (Xu and Shrager, 2005). How early a protein becomes detectable depends not only on the abundance and distribution but also on availability of antigens and on the avidity of the antibodies used. The later can in turn vary depending on the antigen preservation and staining conditions in general. We therefore refer to the lack of detectability of the antigens rather than their absence.

Microscopy. Fluorescence microscopy was performed at the shared microscope facility of the Mount Sinai School of Medicine. Epifluorescent imaging was done with a $40 \times$ objective on a Zeiss (Oberkochen, Germany) Axiophot using Openlab software (Improvision, Lexington, MA).

Confocal imaging was done using the Zeiss 510 system. Using a $40 \times$ objective, three-channel $Z$ stacks of four to six optical sections at $0.45 \mu \mathrm{m}$ intervals were collected in sequential mode. Single optical sections were typically exported as TIFFs from within the $Z$ stack and further processed in Photoshop 7.0 (Adobe Systems, San Jose, CA). Images used for comparison of detectability were acquired and processed using the same settings.

Image analysis. The distribution of $\mathrm{NF}$, ankG, and MAP2 was quantified using line intensity scans on raw 8- or 12-bit images using a onepixel-wide line in NIH ImageJ. The intensity values obtained were imported into Excel (Microsoft, Seattle, WA) and averaged across 20-pixel intervals before plotting. Background traces were obtained on cell-free regions of the coverslips for comparison. Identically acquired images were used when the same markers were compared.

\section{Particle tracking}

Laser tweezers and video microscopy. Single-particle tracking was performed on a custom modified Zeiss Axiovert 200 inverted fluorescence microscope. Modifications included the replacement of the specimen stage with a electronically steerable specimen stage (up to $200 \mu \mathrm{m} / \mathrm{s}, x-y$; $1 \mu \mathrm{m} / \mathrm{s}, z$ ) (Cell Robotics, Albuquerque, NM), the addition of a CCD video camera to the side port (Hamamatsu, Bridgewater, NJ), a PMDIC system, and the addition of a $1.5 \mathrm{~W} \mathrm{Nd:YAG} \mathrm{continuous} \mathrm{wave} \mathrm{laser}$ operating at $1064 \mathrm{~nm}$ to the same light path as the fluorescence excitation light. An objective heater kept neuron chambers at $36^{\circ} \mathrm{C}$.
Laser tweezer experiments were performed with the laser focused through a $100 \times$ oil immersion objective lens. For bead-binding assays, beads were held down for 10-30 s on the cell surface from the top using a $0.81 \mathrm{pN}$ optical-gradient laser trap. Any bead that stayed in contact with the cell for at least $30 \mathrm{~s}$ after deactivation of the trap was scored as attached. Cells were photographed for later recognition. After laser tweezer experiments, the survival of the cell was monitored by surveillance of growth cone movement or observation of intracellular traffic. Only cells that survived the procedure were scored. Antibody-coupled beads bound poorly ( $10 \%, 4$ of 40$)$ to HeLa cells not expressing the antigen. Antibodycoupled beads bound well $(>67.5 \%)$ to neurons. Protein A-coupled beads bound poorly (6\%) to neurons. The movement of attached beads was recorded on S-VHS tapes (Maxell, Fairlawn, NJ) using a DS555 video recorder (Panasonic, Secaucus, NJ). Videos were recorded a frequency of 30 frames per second. The images were modified using an Argus 20 image processor, depending on the quality desired, background subtraction, or PMDIC were applied.

Data processing. S-VHS videos of diffusing beads were digitized using Isee software (Inovision, Cleveland, $\mathrm{OH}$ ), and $x$ and $y$ bead coordinates were determined from video micrographs using single-particle-tracking routines performed using Isee software (Inovision) running on a Unix workstation. Tracking accuracy was $5-10 \mathrm{~nm}$ for $1 \mu \mathrm{m}$ beads. The acquired data were processed using Excel software (Microsoft). Data analysis means were provided to us by Dr. Dan Felsenfeld. Alpha values were determined as by Feder et al. (1996).

\section{Generation of constructs}

Extracellular domain NF fusion protein. The extracellular domain of neurofascin (amino acids 34-1043) was cloned into pET102/D-TOPO vector (Invitrogen) creating a $6 \times$-His-tagged protein. The expression of the fusion protein was induced in BL21 cells, according to the instructions of the manufacturer, and cell lysates were run on SDS-PAGE for Western blots.

NgCAM and NF FIGQY mutant construction. Full-length wild-type $\mathrm{NgCAM}$ was cloned into vector pcDNA1.1 (Invitrogen) at HindIII site to obtain pcDNA1NgCAM. For generation of NgCAM mutants, sitedirected QuikChange mutagenesis kit (Stratagene, La Jolla, CA) was used to generate point mutation at position 1258 of NgCAM, in which tyrosine residue was substituted with alanine, phenylalanine, and aspartic acid, respectively, and then subcloned into pcDNA1 vector at HindIII$\mathrm{XbaI}$ sites. Similar mutagenesis procedure was applied to generate neurofascin mutants with substitution of tyrosine residue at position 1146 with the respective amino acid residue described above. All of the mutants were cloned into vector $\mathrm{pCB} 6 \mathrm{BS}$ at $\mathrm{XhoI}-\mathrm{XbaI}$ sites, which was used for expression in mammalian cells.

\section{Results}

\section{Cultured hippocampal neurons are a good model system for studying IS assembly}

We first tested whether cultured hippocampal neurons could serve as a good model system to study axonal compartmentalization and IS formation by determining the localization of known IS components. ankG and NF were found highly enriched in the IS of mature [10 d in vitro (DIV)] neurons (Fig. $1 A-C$ ) (for antibody characterization, see supplemental Fig. 1, available at www.jneurosci.org as supplemental material). MAP2 was used to visualize soma and dendrites of mature neurons (Fig. $1 A-C$, blue). The IS, as marked by ankG staining, was tightly compacted and measured $\sim 45-60 \mu \mathrm{m}$ of the length of the axon. Fluorescence intensity scans of mature cells stained simultaneously with antibodies against ankG and NF showed that the distribution of the two proteins in the IS closely paralleled each other (Fig. 1C,D).

To determine at what stage of axonogenesis the ankG-positive IS was assembled, cultures at DIV3-DIV7 were stained with antibodies against ankG and MAP2, and the percentage of cells with detectable accumulation of ankG at the IS was determined. The percentage of ankG-positive cells at early days of culture was 

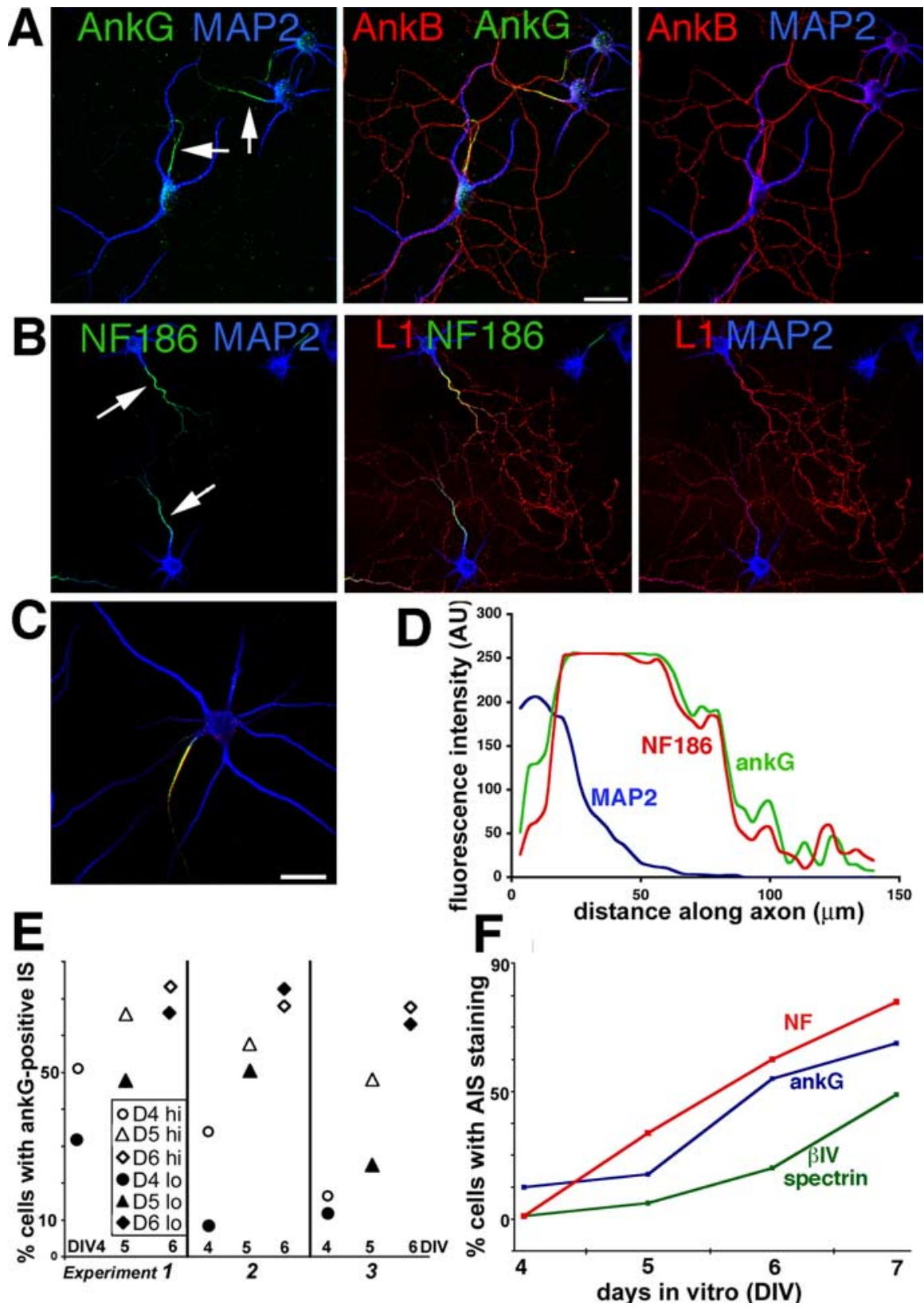

Figure 1. Differential distribution of $\mathrm{L} 1$ family members and ankyrins in cultured hippocampal neurons. $\boldsymbol{A}, \boldsymbol{B}, \mathrm{Hippocampal}$ neurons cultured in vitro for 10-12 d (DIV) were stained with antibodies against the L1 family members $L 1$ (red) and neurofascin (green) $(\boldsymbol{B})$ and against ankyrin (green) and ankyrin B (red) $(\boldsymbol{A})$. MAP2 was counterstained in blue to indicate the location of soma and dendrites. ankB and L1 are found enriched along axons, whereas ankG and NF are found enriched on the axonal IS (see arrows). C, D, The staining intensity of cells triple stained with antibodies against MAP2 (blue), NF (red), and ankG (green) were analyzed by line intensity scans using NIH ImageJ software. A typical cell is shown. Tracings were started at the soma/hillock boundary and extended for 80-100 $\mu \mathrm{m}$ along the axon. The intensity of NF (red profile) and ankG (green profile) closely align along axons of stage 4 neurons. The IS, as delineated by ankG and NF staining, measures $\sim 60 \mu \mathrm{m}$. MAP2 (blue profile) staining, in contrast, falls off sharply at the axon hillock. Scale bars, $50 \mu \mathrm{m}$. $\boldsymbol{E}$, Density of neuronal cultures influences the time course of ankG accumulation at the IS. Cultures were plated in $60 \mathrm{~mm}$ dishes containing several coverslips at two different densities [hi, 200,000 cells per $60 \mathrm{~mm}$ dish (open symbols); 10, 100,000 cells per $60 \mathrm{~mm}$ dish (filled symbols)], and one coverslip was removed from the same dish at consecutive days (DIV4, circles; DIV5, triangles; DIV6, diamonds) and stained against ankG. The results are shown for three independent cultures. Despite variability, the low-density (filled symbols) cultures showed slower maturation of the IS in each experiment than the higher-density cultures (open symbols). $\boldsymbol{F}$, Coverslips were fixed at consecutive days from the same culture and stained against NF (red), ankG (blue), and $\beta$ IV-spectrin (green). The percentage of cells showing IS enrichment is shown for one such experiment.

variable. To determine whether cell density correlated with the accumulation of detectable ankG at the IS, we plated cells at two different densities (low, 100,000 cells per $60 \mathrm{~mm}$ dish; high, 200,000 cells per $60 \mathrm{~mm}$ dish) in three separate experiments and determined the percentage of ankG-positive cells between DIV4 and DIV6. In each high-/lowdensity paired culture, the higher-density dishes showed a higher percentage of ankGpositive cells than the lower-density dishes. The difference tended to be most pronounced at DIV4 and disappear by DIV6 (Fig. 1E). We used lower-density cultures for these studies because they allowed studying the assembly of the IS in a longer time window. Other IS-resident proteins accumulated in the IS with a similar time course to ankG (Fig. $1 F$ ) (supplemental Fig. 2, available at www.jneurosci.org as supplemental material).

\section{Immature axons progressively} accumulate initial segment components We characterized the development of the IS during axonogenesis using immunofluorescence against IS resident proteins. Based on the staining patterns of ankG, $\mathrm{NF}$, and MAP2, we classified three phases of IS maturation, named IS1, IS2, and IS3. IS3 is the mature pattern, as shown in Figure 1. Before axonogenesis [stage 2 (staging according to Dotti et al., 1988)], neither neurofascin nor ankG could be reliably detected (data not shown). After the axon started to grow (stage 3), ankG and NF became increasingly enriched at the IS (Fig. 2). Cells with no detectable accumulation of ankG at the IS were categorized as IS1, the least mature phase of stage 3 cells. In most cells at this stage, ankG staining was not detectable anywhere (Fig. $2 A, C$, middle). MAP2 staining was moderately bright in IS1 cells and was not yet excluded from the longest process (Fig. 2, blue). Using several antibodies raised against the mucin-rich domain of NF186 as well as an antibody directed against a non-mucin-rich domain epitope of NF, we found that NF staining in IS1 cells was often weak/nondetectable. We additionally observed cells in which NF staining was detected enriched along the axon and less bright on dendrites (Fig. $2 A, B$, right). This broader NF staining was observed in a subset of cells and represented a transient stage.

We then determined the distribution of the related proteins $\mathrm{L} 1$ and ankB. Both L1 and ankB were found enriched on axons in mature (IS3) cultured hippocampal neurons (Fig. $1 A, B$, red), similarly to their in vivo distribution (Scotland et al., 1998). In IS1 cells, both L1 and ankB were already enriched in axons and did not accumulate significantly in dendrites (Fig. $2 B$, middle, $C$, right). Both $\mathrm{L} 1$ and NF are therefore preferentially enriched on axons, as soon as axons can be distinguished morphologically (stage 3 ). 

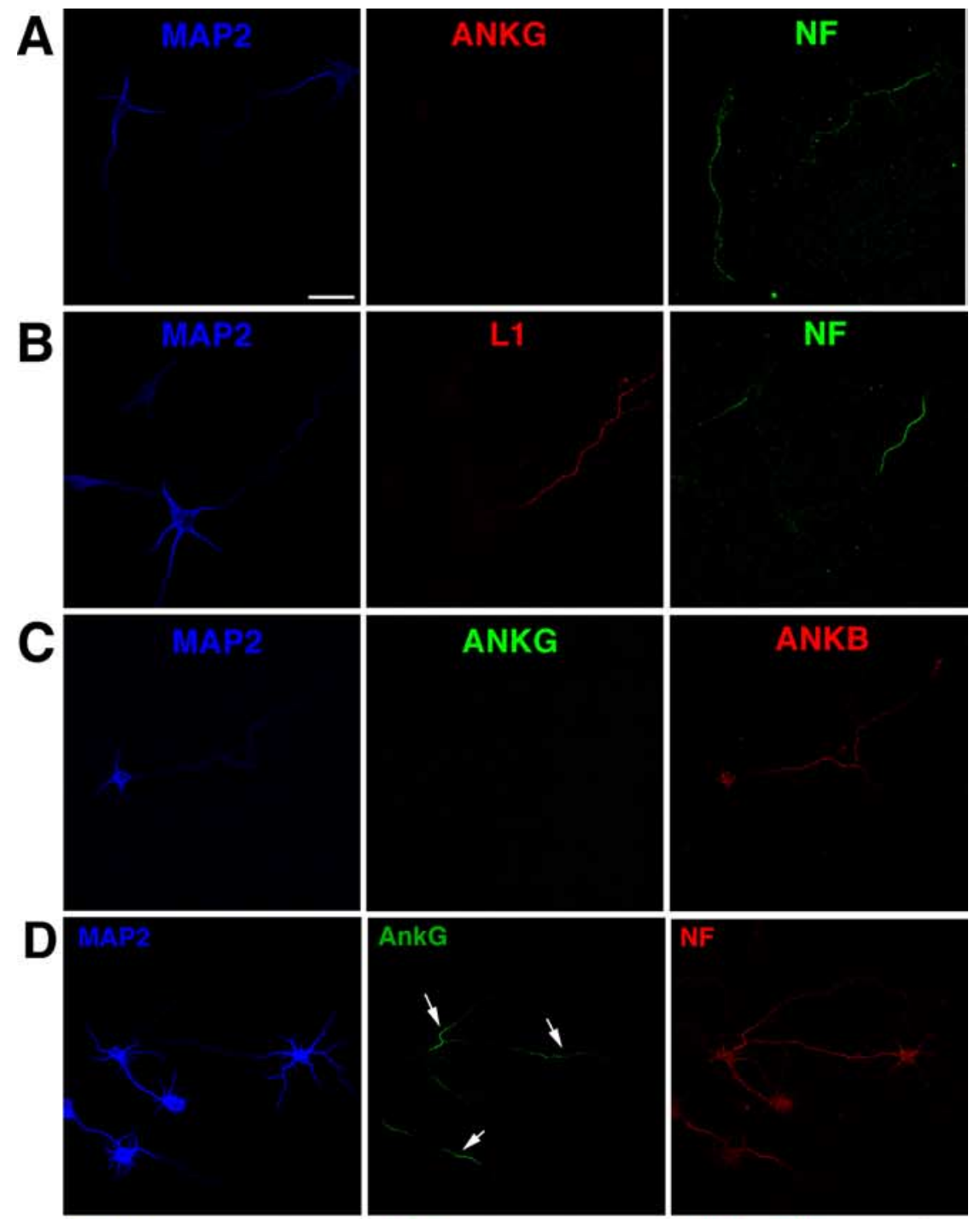

D

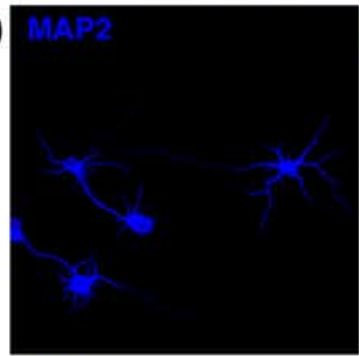

E
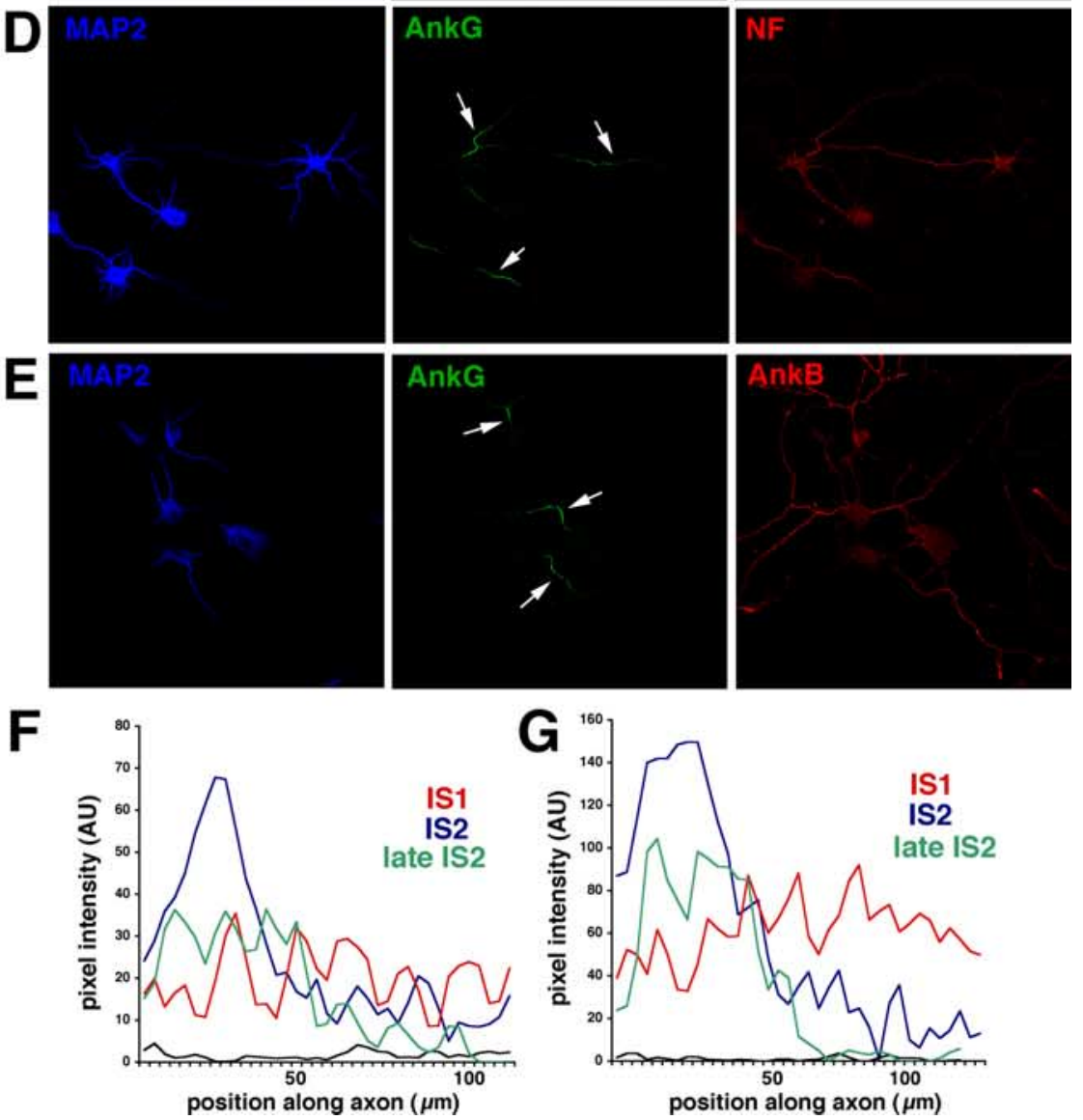

Figure 2. Expression of L1 family members and ankyrins during axonogenesis. $\boldsymbol{A}-\boldsymbol{C}$, Localization of $\mathrm{L} 1 \mathrm{family}$ members and ankyrins in early stage 3 neurons before detectable ankG accumulation at the IS (equivalent to IS1 stage). Early stage 3 neurons were stained with antibodies against MAP2 (blue), ankG (red), and NF (green) (A), against MAP2 (blue), L1 (red), and NF (green) (B), and against MAP2 (blue), ankG (green), and ankB (red) (C). MAP2 staining in IS1 cells is bright in the somatodendritic domain
IS1 swiftly gave way to IS2, in which ankG was detectably enriched at the IS of rapidly outgrowing axons. During IS2, the enrichment of IS components in the proximal portion of the axon became more pronounced and more tightly localized. In early IS2, MAP2 staining was enriched in the somatodendritic domain but tapered into the proximal portion of the axon (Fig. $2 D, E$, middle, arrows). AnkG at this stage was found clearly enriched at the initial part of the axon (Fig. 2D,E, middle). The lengths of these young ISs were 45-60 $\mu \mathrm{m}$, not significantly different from mature ISs. NF primarily codistributed with ankG, also showing highest intensity on the IS and tapering to the more distal portion of the axon in which it was still clearly detectable (Fig. 2D, right). As in IS1, ankB immunoreactivity was more pronounced and still axonally enriched at IS2 (Fig. 2E, right). In late IS2 cells, anti-ankG patterns was clearly restricted to the proximal portion of the IS and rapidly diminished in intensity at the proximal and distal margins. Again, NF immunostaining closely paralleled ankG distribution. Figure 2, F and $G$, shows intensity line scans from examples of IS1, IS2, and late IS2 cells taken from the same microscope field. Both NF (Fig. 2F) and ankG (Fig. 2G) became enriched at the IS coordinately. As the staining intensity for NF became stronger on the IS, it became weaker on the distal axon.

\section{AnkyrinG expression on the IS}

correlates with low diffusibility of NF

Several IS-resident proteins (including NF) lose IS localization in ankG-deficient neurons (Zhou et al., 1998; Jenkins and Bennett, 2001; Pan et al., 2006). This loss of proper accumulation at the IS could be

$\leftarrow$

but is still detectable a significant distance into the axon. ankG is not detectable in the IS. NF, L1, and ankB are all found axonally enriched. NF staining extends past the initial segment. IS1 can therefore be classified based on MAP2 and NF staining or on MAP2 and ankG staining. $\mathbf{D}-\mathbf{G}$, Localization of $L 1$ family members and ankyrins in late stage 3 neurons after ankG becomes IS-enriched (equivalent to stage IS2). D, Late stage 3 neurons were stained against MAP2 (blue), ankG (green), and NF (red). $E$, Late stage 3 neurons were stained against MAP2 (blue), ankG (green), and ankB (red). Arrows point at the IS. Scale bar, $50 \mu \mathrm{m} . \boldsymbol{F}, \mathbf{G}$, Intensity line scans from three DIV4 cells stained against NF $(\boldsymbol{F})$ and ankG $(\boldsymbol{G})$. Scans from cells classified as IS1 (red), IS2 (blue), and late IS2 (green) by their ankG patterns were plotted in the same graph for comparison. The intensity of staining increases at the IS and ultimately sharpens into a wide plateau with sharp rise and fall transitions at the borders. At the same time, the staining in the distal axon diminishes. All images were photographed and processed identically. The black line shows the level of nonspecific background staining. 

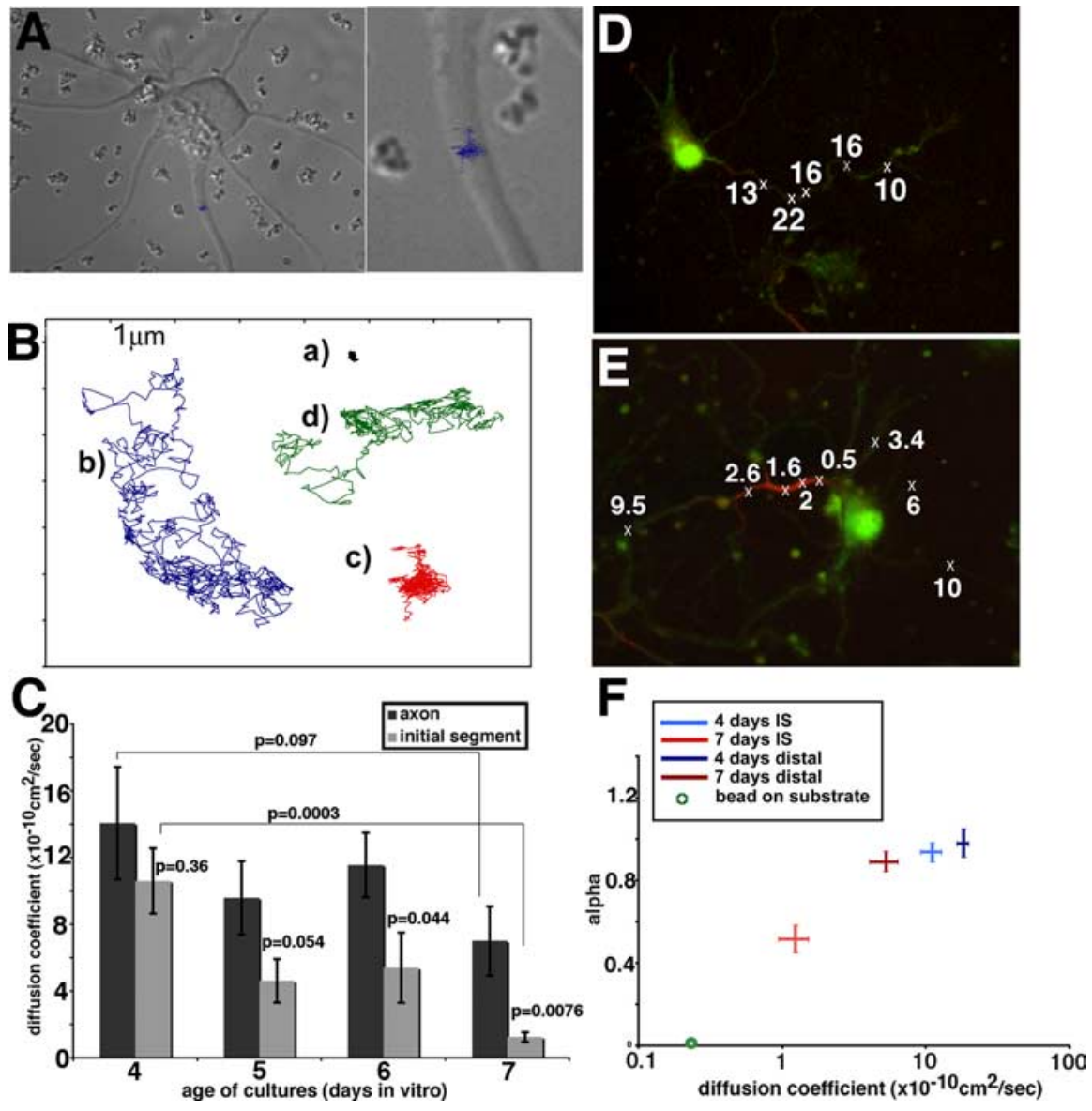

Figure 3. Low diffusibility of NF correlates in time and space with ankG expression in the IS. $A$, A cultured neuron used for single-particle tracking with anti-NF-coated beads. The trajectory traversed by one bead over a 30 s time course ( 900 video frames) is superimposed on the differential interference contrast image of the cell. This bead was placed on the IS and did not diffuse far along the axon but stayed in a confined area. The right is a close-up. B, Examples of bead trajectories obtained with anti-NFcoupled beads. a, Stationary bead; $b$, freely diffusing bead; $c$, confined bead; $d$, combination of short confinement phases and free diffusion. $C$, Diffusion coefficients $\left(D_{2}-D_{4}\right)$ were calculated from mean square displacement plots for anti-NF-coupled beads measured on cultured neurons of days 4-7. The diffusion coefficients were determined for beads initially placed on the IS (gray bars) or the distal axon (black bars). $D_{2}-D_{4}$ indicates essentially unrestricted diffusion for beads on DIV4 regardless of location. At later ages, beads placed on the distal axon diffused more rapidly than beads placed on the IS. Error bars indicate SEM. $p$ values were calculated between pairs as indicated by the brackets. $\boldsymbol{D}, \boldsymbol{E}$, Bead diffusion was measured on DIV6 neurons. Subsequently, cells were fixed and stained with an antibody against ankG (red). The original cells were relocated, and bead position and behavior were compared with ankG staining. Crosses indicate position of beads placed. Numbers indicate $D_{2}-D_{4}\left(\times 10^{-10} \mathrm{~cm}^{2} / \mathrm{s}\right)$. Beads overlying the ankG-positive segment (red) diffused more slowly than beads overlying the ankG-negative axon segments. $\boldsymbol{F}$, The diffusion mode of beads was analyzed by determining $\alpha$ in curve fit analysis of the MSD plots for each bead. A linear MSD plot results in an $\alpha$ of 1, indicative of free Brownian diffusion. $\alpha$ values of less than one are indicative of confined diffusion mode. The diffusion coefficients $\mathrm{D}_{2}-\mathrm{D}_{4}$ are plotted on the $x$-axis, and $\alpha$ is plotted on the $y$-axis. The intersection of the lines corresponds to the average value for each condition, whereas the lengths of the arms of each cross indicate the SEM. The averages and SEM are shown for beads at DIV4 placed on the IS (light blue), DIV4 on the distal axon (dark blue), DIV7 on the IS (red), DIV7 on the distal axon (brown), and beads immobile on the substrate (green circle).

attributable to a requirement for ankG in secretory delivery to the IS (i.e., targeting), for tethering/retention in the plasma membrane, or for both. To acutely assay retention, we used two experimental strategies that measure different aspects of the behavior of the surface pool of membrane proteins: diffusion measurements and detergent extractions of live cells (Winckler et al., 1999). Several membrane proteins show low diffusibility in the IS of cultured hippocampal neurons (Winckler et al., 1999; Nakada et al., 2003). Several mechanisms can cause low diffusibility. The first is direct binding/tethering to membrane skeleton. Membrane proteins that show low diffusibility attributable to direct tethering also show high resistance to extraction by non-ionic detergents (Winckler et al., 1999). L1 has been shown to fall into this category. Other proteins diffuse slowly but remain highly detergent soluble. These proteins (Thyl is an example) are not directly tethered to cytoskeleton but diffuse slowly because of a high obstacle course of immobile proteins in the membrane restricting their diffusion.

We tested whether the surface pool of $\mathrm{NF}$ at the IS was restricted in its diffusibility, similarly to sodium channels (VGNCs) and other membrane components. To measure diffusion coefficients of NF, we performed single-particle tracking of antibody-coupled beads placed on the axonal surface using optical tweezers (see Materials and Methods). Bound beads were videotaped, and several hundred (up to 1000) consecutive frames were digitized. Bead movements were analyzed using tracking software (Fig. $3 A$ ). Figure $3 B$ shows examples of the kinds of trajectories obtained. The same scale is used for each trajectory. Some beads diffused freely and cover a large area during a 1000-frame time course (trajectories b and d), whereas other beads were mostly stationary (trajectory a) or covered a small confined area (trajectory $\mathrm{c}$ ). The digitized trajectories were used to calculate diffusion coefficients $\left(\mathrm{D}_{2}-\mathrm{D}_{4}\right)$ (Kusumi et al., 1993). Analysis of many beads on cultures between DIV4 and DIV7 showed that NF diffusibility diminished with increasing age (Fig. 3C). In particular, NF showed reduced diffusibility on the IS compared with the more distal portions of the axon in DIV7 cultures $(p=0.0076)$. In younger cultures (DIV4), NF diffusibility was not restricted even in the IS $(p=0.36)$. Diffusion coefficients on the distal axon might also be somewhat slower between DIV4 and DIV7 ( $p=0.097)$.

To test whether the decreased diffusibility of NF correlated with ankG accumulation at the IS, single-particle tracking was performed on several cells from different ages followed by fixation and immunostaining against ankG. The same cells were relocated on the coverslip and photographed. Figure $3 D$ exemplifies a cell in which all beads diffused rapidly. ankG enrichment in the IS was not detectable. The cell shown in Figure 3E, conversely, showed enriched ankG staining on the IS. The four beads directly overlaying ankG staining (see crosses) showed slower diffusion coefficients. The slow diffusibility of NF therefore correlated in time and space with ankG expression in the IS.

We next analyzed the diffusion mode of beads by calculating mean square displacement (MSD) plots. Linear MSD plots indicate free Brownian diffusion $(\alpha=1)$, whereas downward deviation from linearity is indicative of a confined mode of diffusion $(\alpha<1)$ (Feder et al., 1996; Ewers et al., 2005). Nonlinear confined diffusion arises when a bead is confined ("corralled") to a small area within the plane of the membrane (Kusumi et al., 
1998). This means that the trajectory of a bead covers a smaller area over time than would be predicted from its diffusion coefficient. The $\alpha$ coefficient was determined from line fits of each MSD plot in which $r^{2}$ $=4 D t^{\alpha}$. Beads on DIV4 neurons and beads on the distal axon of DIV7 neurons have an average $\alpha$ near 1, indicative of free Brownian motion (Fig. $3 F$ ). The diffusion behavior of IS-placed beads on DIV7 cells, conversely, showed a decrease in $\alpha$, indicating confined mode of motion (Fig. $3 F$ ). Although VGNCs and lipids were reported to become immobilized in the IS by $\mathrm{Na}$ kada et al. (2003), confined diffusion was not reported for either probe. It is unclear whether the low signal-to-noise ratio of single-fluorophore imaging rendered confinement undetectable in the other study, or only immobile and freely mobile probes were recorded with no intermediate behavior.

\section{NF accumulated in the IS is resistant to detergent extraction}

In previous work, we showed that L1 exhibited increased resistance to detergent extraction specifically in the IS in which its diffusibility is low (Winckler et al., 1999). VGNC chimeras containing ankyrin binding sites localize to the IS and similarly show detergent resistance (Garrido et al., 2003). The increased detergent resistance in the IS reflects binding to detergent-resistant elements of the cytoskeleton. Live detergent extractions were therefore used to determine whether the low diffusibility of NF was attributable to direct tethering. To first test that cytoskeletal elements were resistant to detergent extraction, cultured neurons were extracted before fixation in $0.5 \%$ Triton $\mathrm{X}-100$ for $8 \mathrm{~min}$ at $37^{\circ} \mathrm{C}$ and then fixed and stained with antibodies against tubulin, MAP2, and ankG. We find that tubulin (red) and ankG (green) remained associated with the Triton $\mathrm{X}$-100-insoluble cytoskeleton to a high degree (Fig. $4 A^{\prime}, \mathrm{TE}$ ), similar to non-extracted controls (Fig. 4A). MAP2 (blue), conversely, was primarily solubilized under these conditions. Filamentous actin (F-actin) was reported previously to be detergent resistant in the IS (Winckler et al., 1999). To test whether NF was similarly associated with the detergent-resistant cytoskeleton in the IS, we stained unextracted control cultures (Fig. 4B) and extracted cultures (Fig. $4 B^{\prime}$, TE) with antibodies against NF and ankG. NF (red) was stably associated with the IS after detergent extraction and colocalized with detergent-resistant ankG (green). Therefore, NF diffusibility and detergent resistance correlate in time and space with ankG expression.

\section{NF binds preferentially to ankG in HEK 293 cell recruitment assays}

Our results so far suggest that ankyrin tethers and thereby immobilizes and retains NF in the IS. Because ankB is present all along the axon (Fig. 1,2), ankyrin binding per se can only account for IS enrichment if NF shows preferential binding to ankG over ankB. We therefore performed ankyrin recruitment assays in HEK 293 cells, as described previously (Zhang et al., 1998; Gil et al., 2003; Nishimura et al., 2003). HEK 293 cells transfected with ankG-
GFP alone (Fig. 4D) or ankB-GFP alone (Fig. 4C) showed cytosolic GFP fluorescence with dark nuclei excluding the soluble ankyrins. Plasmid levels for ankB-GFP and ankG-GFP were titrated to give similar levels of GFP fluorescence intensity. Under these conditions, the two ankyrins expressed alone behaved similarly and remained cytosolic (Fig. 4G). When HA-NF (Fig. 4 $E^{\prime}$ ) was coexpressed with ankG-GFP (Fig. $4 E$ ), the nuclear exclusion staining was no longer apparent, but rather GFP fluorescence was recruited to the plasma membrane resulting in staining of surface structures such as filopodia and ruffles (Fig. $4 E$ ). A total of $88 \%$ of cells coexpressing HA-NF and ankG-GFP recruited ankG-GFP to the membrane by these criteria. When a 10 -fold range of ankG-GFP plasmid was used in cotransfections with HA-NF, the percentage of recruiting cells ranged from 88 to $94 \%$. When HA-NF was coexpressed with ankB-GFP (Fig. $4 F, F^{\prime}$ ), in contrast, only $21 \%$ of cells recruited ankB-GFP to the plasma membrane (Fig. 4G). If a 10-fold range of ankB-GFP plasmid was used for cotransfections with $\mathrm{HA}-\mathrm{NF}$, the percentage of recruiting cells ranged from 8 to $27 \%$. Therefore, HA-NF shows preferential recruitment of ankG over ankB in HEK 293 cells. This observed preference of $\mathrm{NF}$ for ankG could account for the slow diffusion and detergent resistance of NF in the IS.

\section{Ankyrin-binding of L1/NgCAM is not necessary for axonal targeting}

L1 did not colocalize with ankG at the IS in unextracted control cells (Fig. $5 A$, left) but rather codistributed with ankB all along axons. After extraction, in contrast, L1 was preferentially detergent resistant exactly in the same places in which ankG was expressed (Fig. 5B, TE, right) and showed greatly diminished retention in the distal axon in which ankB was expressed, confirming our previous work (Winckler et al., 1999). We therefore tested 

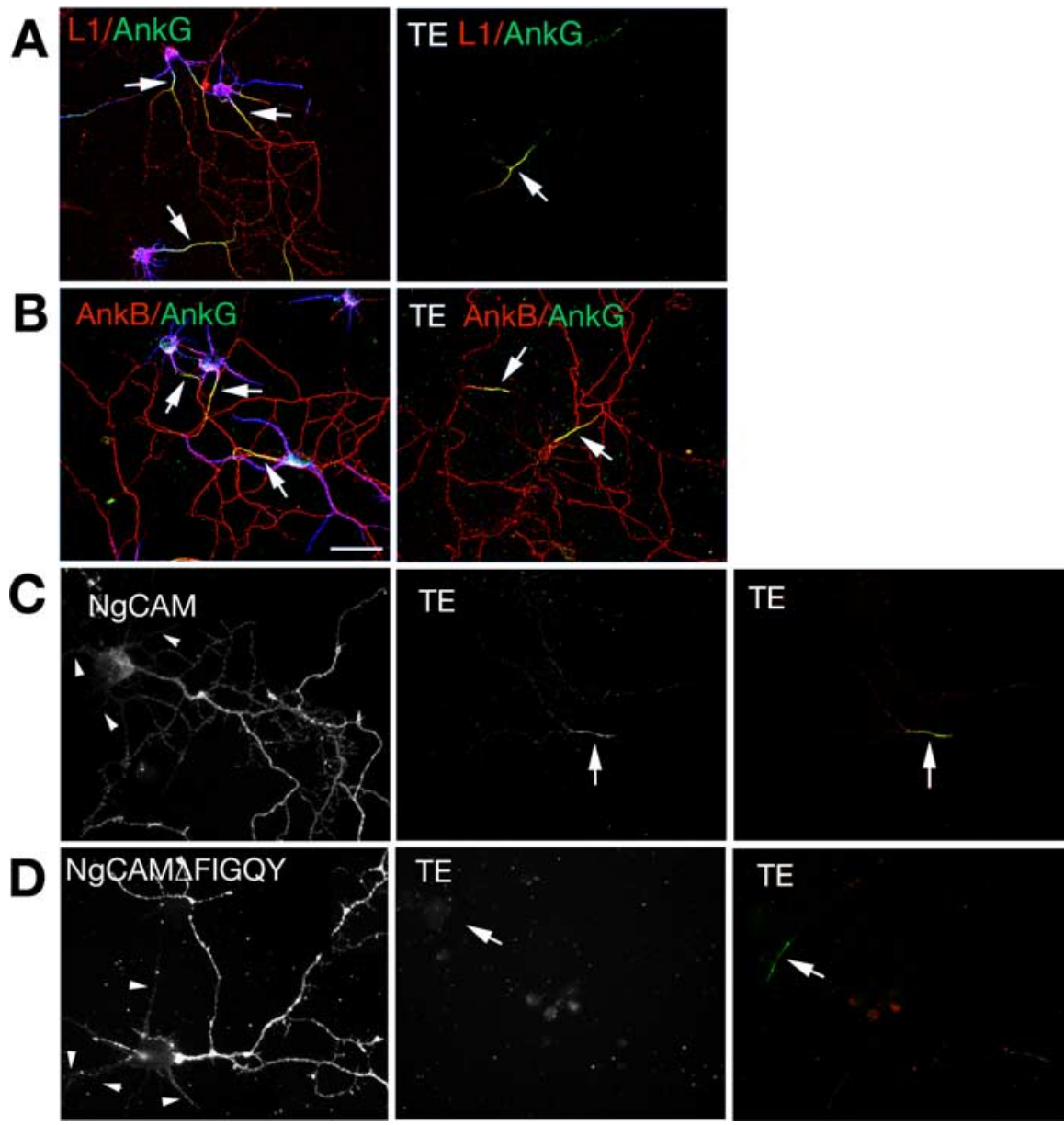

Figure 5. The FIGQY motif is required for IS detergent resistance of $\mathrm{L} 1 / \mathrm{NgCAM} . A, B$, Localization of $\mathrm{L} 1$ (red in $A$ ), ankG (green), and ankB (red in $\boldsymbol{B}$ ) in non-extracted control neurons (left) and after Triton X-100 extraction (TE) (right). MAP2 staining is in blue. IS are indicated by arrows. Scale bar, $50 \mu \mathrm{m}$. C, D, Localization of $\operatorname{NgCAM}(\boldsymbol{C})$ and $\mathrm{NgCAM} \Delta \mathrm{FIGQY}(\boldsymbol{D})$ in nonextracted control neurons (left) and Triton X-100-extracted (TE) neurons (middle). Anti-NgCAM staining (red) is overlaid over ankG (green) in the right panels to indicate position of ankG-positive IS (arrows). Dendrites are indicated with arrowheads.

whether ankB showed preferential detergent resistance in the IS. Unlike L1, ankB was not retained specifically at the IS. Although not excluded from the IS, ankB was detectable to various degrees along the entire length of axons after detergent extraction (Fig. $5 B)$. Therefore, $\mathrm{L} 1$ and ankB show different extractability patterns in axons.

Given the discrepancy between the steady-state localization of L1 without extraction (all along the axon) and the detergent-resistant L1 pattern (IS-enriched), we wondered whether ankyrin binding was required for axonal accumulation and/or detergent resistance of $\mathrm{L} 1$ at the IS. The FIGQY motif in L1 family members is responsible for binding to ankyrin (Hortsch et al., 1998; Zhang et al., 1998). We therefore created ankyrin binding-defective mutants of NgCAM by either deleting the FIGQY motif (NgCAM $\triangle$ FIGQY) or by mutating the critical tyrosine to alanine (NgCAM FIGQA). NgCAM and its mutants were expressed in cultured hippocampal neurons and detected 18-24 h later with an NgCAM-specific antibody. NgCAM $\triangle$ FIGQY (Fig. 5D) as well as NgCAM FIGQA (data not shown) were localized highly enriched to the axon, indistinguishable from wild-type NgCAM (Fig. 5C). To quantify the extent of polarized expression, we determined the average pixel intensity along axons and along dendrites and calculated an axon/dendrite polarity index (A/D PI) by dividing the average axon intensity by the average dendrite intensity (as in the study by Wisco et al., 2003). The A/D PI for NgCAM was 6.0 (Wisco et al., 2003). NgCAM FIGQA had a similarly high A/D PI of 5.6. Ankyrin binding is therefore dispensable for axonal targeting of $\mathrm{NgCAM}$.

After detergent extraction, no staining was detectable for NgCAMAFIGQY, whereas wild-type $\mathrm{NgCAM}$ was detectably retained in the IS (Fig. $5 C, D$, TE), similarly to endogenous L1. To quantify this observation, coverslips were transfected in parallel with either NgCAM or NgCAM $\triangle$ FIGQY, and the number of transfected cells was counted in unextracted cells and compared with the number of cells showing IS retention after detergent extraction of a coverslip transfected in parallel (Table 1). Similar to NgCAM $\Delta$ FIGQY, NgCAM FIGQA was not retained in the IS after detergent extraction (Table 1). Therefore, the ankyrin-binding domain of $\mathrm{NgCAM}$ was required for IS tethering. Axonal targeting, in contrast, was preserved in the absence of ankyrin binding capacity.

\section{The ankyrin-binding domain of NF is required for IS retention but not for axonal enrichment}

We next asked whether IS accumulation of NF was ankyrin dependent by mutating the FIGQY motif in an HA-tagged version of NF (HA-NF FIGQA). Next, HA-NF and HA-NF FIGQA were expressed in cultured neurons and cells stained with an antibody to the extracellular HA epitope tag. HA-NF was highly enriched on the IS with low levels of expression along the rest of the axon (Fig. 6A). HA-NF FIGQA, conversely, was found all along the axon and did not show IS enrichment (Fig. $6 B$ ). We calculated the enrichment of the NF constructs in the IS relative to dendrites by determining the IS/dendrite polarity index (IS/D PI). The position of the IS was determined by counterstaining with ankG. HA-NF had an IS/D PI of $4.0 \pm 0.4$ (mean \pm SEM), whereas HA-NF FIGQA had an IS/D PI of $1.65 \pm 0.09$ (mean \pm SEM) (Fig. 6E). Two other point mutations in the FIGQY motif (HA-NF FIGQD and HA-NF FIGQF) also essentially lost IS accumulation (data not shown). Therefore, the ankyrin-binding motif of NF was necessary for correct IS localization of HA-NF.

We then tested whether the FIGQY motif mediated tethering of NF in the IS by using live detergent extractions. After detergent extraction, HA-NF staining was still present at the IS (Fig. 6C), similar to endogenous NF. In contrast, no staining against HA-NF FIGQA, HA-NF FIGQD, or HA-NF FIGQF could be detected in either the IS or elsewhere (Fig. 6D, Table 1) (supplemental Fig. 3, available at www.jneurosci.org as supplemental material). Therefore, the FIGQY motif mediates tethering of NF in the IS.

Interestingly, although HA-NF FIGQA failed to accumulate at the IS, it accumulated to high levels on the axonal plasma membrane distally but was found at low levels on dendrites (Fig. $6 \mathrm{~B}$, arrows). We quantified its localization by determining $\mathrm{A} / \mathrm{D}$ PI as before. As a control, we expressed a membrane protein lacking its cytoplasmic tail $(\mathrm{CD} 4 \Delta \mathrm{CT})$. CD4 $4 \mathrm{CT}$ was present on 
Table 1. Detergent resistance at the IS is mediated by the FIGQY motif

\begin{tabular}{|c|c|c|c|c|c|c|}
\hline Construct & $\begin{array}{l}\text { Exp. 1, } \\
\text { No. of transfected cells } \\
\text { on nonextracted } \\
\text { coverslip }\end{array}$ & $\begin{array}{l}\text { Exp. 1, } \\
\text { No. of cells with axonal } \\
\text { IS retention on } \\
\text { extracted coverslip }\end{array}$ & $\begin{array}{l}\text { Exp. 2, } \\
\text { No. of transfected cells } \\
\text { on nonextracted } \\
\text { coverslip }\end{array}$ & $\begin{array}{l}\text { Exp. 2, } \\
\text { No. of cells with axonal } \\
\text { IS retention on } \\
\text { extracted coverslip }\end{array}$ & $\begin{array}{l}\text { Exp. } 3 \text {, } \\
\text { No. of transfected cells } \\
\text { on nonextracted } \\
\text { coverslip }\end{array}$ & $\begin{array}{l}\text { Exp. } 3 \text {, } \\
\text { No. of cells with axonal IS } \\
\text { retention on extracted } \\
\text { coverslip }\end{array}$ \\
\hline $\mathrm{NgCAM}$ & 70 & 23 & 60 & 23 & 68 & 21 \\
\hline $\mathrm{NgCAM} F \mid G Q A$ & 71 & 0 & 13 & 0 & 57 & 0 \\
\hline $\mathrm{NgCAM} \mathrm{FIGQD}$ & 56 & 6 & 13 & 0 & 48 & 3 \\
\hline $\mathrm{NgCAM} \mathrm{FIGQF}$ & 63 & 0 & 46 & 0 & 59 & 1 \\
\hline $\mathrm{HA}-\mathrm{NF}$ & 132 & 190 & 124 & 141 & 187 & 99 \\
\hline HA-NF FIGQA & 112 & 1 & 70 & 0 & 92 & 0 \\
\hline $\mathrm{HA}-\mathrm{NF}$ FIGQD & 124 & 3 & 109 & 0 & 37 & 0 \\
\hline HA-NF FIGQF & 199 & 0 & 70 & 1 & 114 & 0 \\
\hline myc-L1 & 38 & 22 & 21 & 16 & & \\
\hline myc-L1 FIGQF & 52 & 0 & 72 & 0 & & \\
\hline myc-L1 FIGQH & 49 & 0 & 68 & 1 & & \\
\hline
\end{tabular}

Exp., Experiment; No., number.

both axons and dendrites with an A/D PI of $1.4 \pm 0.14$ (mean \pm SEM). HA-NF had an A/D PI of $1.9 \pm 0.26$ (mean \pm SEM), showing no significant accumulation on the distal axon past the IS (Fig. 6 E). The A/D PI of HA-NF FIGQA, in contrast, was $3.8 \pm$ 0.25 (mean \pm SEM), demonstrating significant axonal enrichment. Loss of IS localization of NF FIGQA was reported previously (Lemaillet et al., 2003), but axonal enrichment of the mutant was not noted. This might be attributable to the younger age of the neurons used in the other study. We conclude that axonal enrichment and IS retention of NF were separable: only IS accumulation of HA-NF required the FIGQY motif, whereas axonal targeting did not.

\section{Phospho-FIGQY forms of L1 family members are spatially segregated}

Although L1 codistributed with ankB throughout the axon, it was highly diffusible and detergent soluble on the distal parts of the axon, suggesting that it was generally not tethered and retained by binding to ankB. Previous work by Bennett's group showed that the tyrosine of the FIGQY motif of NF could be phosphorylated in vivo and that phosphorylation greatly diminished ankyrin binding (Garver et al., 1997; Tuvia et al., 1997; Jenkins et al., 2001). We wondered whether the spatial differences in L1 behavior could be attributable to differential phosphorylation of the FIGQY motif in different regions of the axon. We used an antibody raised by Bennett and coworkers against the phosphoFIGQY peptide region of NF (Jenkins et al., 2001). This antibody also recognizes the phospho-FIGQY peptide from L1 with lower affinity. We first determined the temporal and spatial expression of phospho-FIGQY forms of L1-CAMs. Neurons between DIV4 and DIV8 were costained with antibodies against ankG, MAP2, and phospho-FIGQY. In IS1 cells (i.e., no accumulation of ankG or NF at the IS), phospho-FIGQY staining was found uniformly on all processes or uniformly on the whole axon (Fig. 7Aa, single channels are shown in $A^{\prime}$ ). In IS2 cells, axonal staining was frequently high with less bright/nondetectable staining at the IS and at growth cones (termed "axon trunk" staining) (Fig. 7Ab). The diminished anti-phospho-FIGQY staining at growth cones can be appreciated better when cells were costained against ankB (Fig. $7 B$ ) because ankB staining was well detectable in growth cones (red; arrows) whereas phospho-FIGQY staining was not (green). In IS2 cells (abundant in DIV6 cells in this culture), axon trunk staining was the most prevalent class (Fig. 7E). In IS3 cells (DIV8), axon trunk staining was still found in $30 \%$ of cells (Fig. $7 A d, E)$. Interestingly, a novel class of phospho-FIGQY staining pattern appeared at this stage ( $\sim 20 \%$ of cells) in which the staining coincided with the IS (Fig. 7E). In older cultures, the phospho-FIGQY staining became progressively weaker, and ultimately virtually no staining was detectable (data not shown).

The distribution patterns of the phospho-FIGQY-containing L1 family members suggest a model to explain the high diffusibility and detergent solubility of L1 on the distal axon as opposed to the low diffusibility and high detergent resistance of L1 and NF in the IS: L1 and NF found at the IS are not phosphorylated on the FIGQY motif and therefore able to bind and be immobilized by ankG (or possibly ankB as well). L1 (and low levels of NF) found in more distal parts of the axon, conversely, are phosphorylated on the FIGQY motif and do not tightly bind ankB, thereby diffusing more rapidly. This model predicts that the phosphoFIGQY forms should be detergent soluble. As predicted, phospho-FIGQY staining was not detergent resistant along the axon (Fig. 7C, green) in which ankB primarily remained (Fig. 7C, red). Interestingly, in occasional cells in which phospho-FIGQY was detected after extraction, it showed IS enrichment (data not shown). We wondered whether in older cells in which phosphoFIGQY staining was no longer detectable, L1 might show increased detergent resistance all along the axon. We indeed found comparable levels of L1 staining retained in the IS and the distal axon in older cells (Fig. 7D, bottom), in contrast to young cells in which detergent resistance of L1 was highest in the IS (Fig. 7D, top). We propose that diffusional and extraction behavior of surface L1 in hippocampal cultures is attributable to spatial and temporal regulation of its ankyrin binding capacity.

\section{Discussion}

\section{Role for ankyrin in retention of neurofascin at the IS plasma membrane}

In ankG knock-out neurons (Zhou et al., 1998; Jenkins and Bennett, 2001), NF accumulation at the IS is lost, suggesting a central role for ankG in IS formation and/or stabilization in cultured neurons. In these long-term experiments, a secretory targeting role and a post-insertion retention role for ankG cannot be distinguished. We present several pieces of evidence for a retention role of ankG at the IS. First, restricted diffusion of NF correlates in time and space with ankG expression. Second, restriction of NF to the IS is compromised when the ankyrin-binding motif is mutated. Third, NF is detergent resistant at the IS, but ankyrin-binding mutants of NF lose detergent resistance. Our finding that NF shows preferential binding to ankG in HEK 293 cells presents a likely mechanism for selective retention of NF at the IS versus the rest of the axon. These observations show 

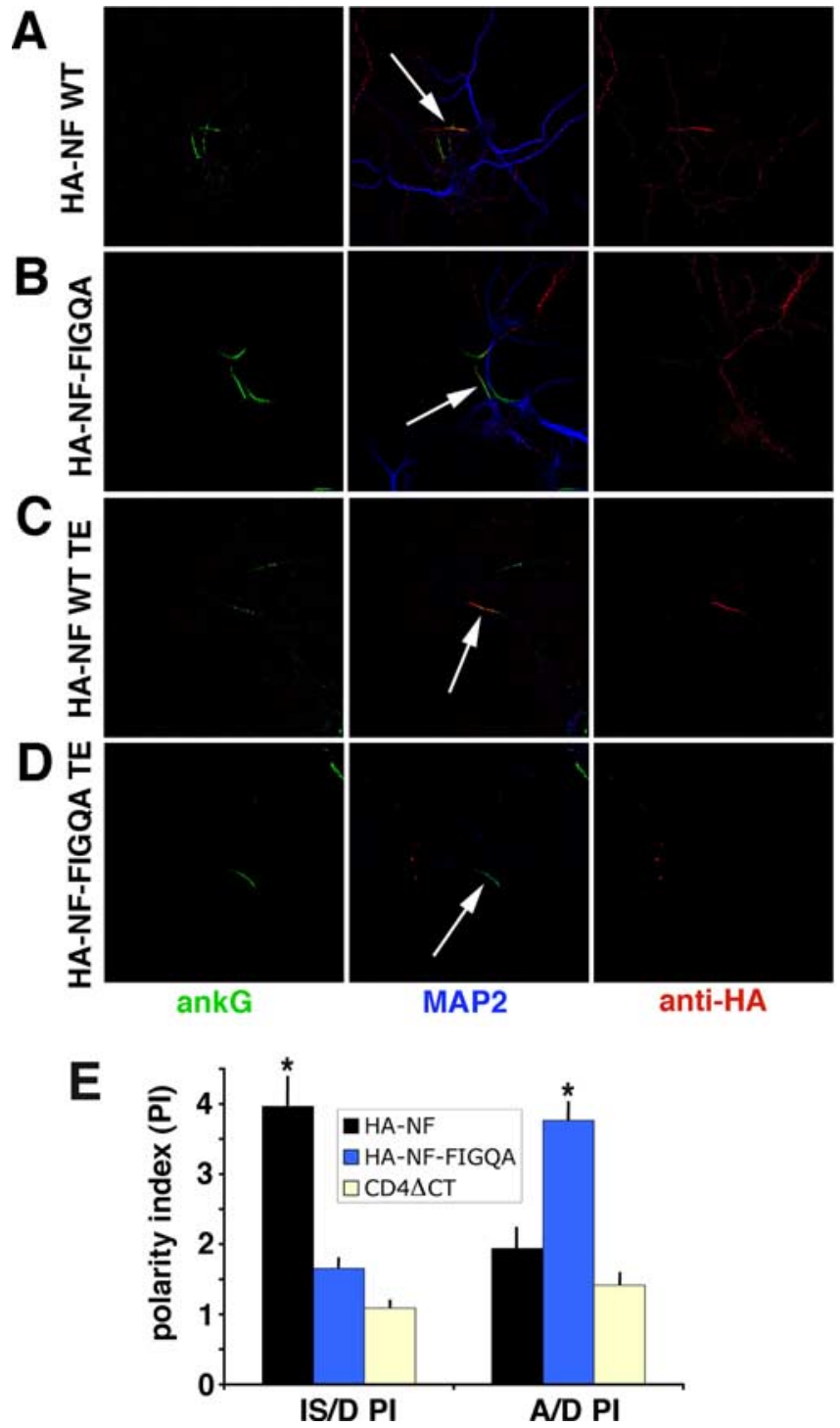

Figure 6. Mutation of the FIGQY motif of NF impairs IS localization and retention. $\boldsymbol{A}-\boldsymbol{D}$, NF-HA $(\boldsymbol{A}, \boldsymbol{C})$ or HA-NF FIGQA $(\boldsymbol{B}, \boldsymbol{D})$ were expressed in hippocampal neurons and either fixed directly $(\boldsymbol{A}, \boldsymbol{B})$ or fixed after live Triton X-100 extraction (TE; $\boldsymbol{C}, \boldsymbol{D})$. Anti-HA staining is visualized in red, ankG staining in green, and MAP2 staining in blue. Single red channel images (right), single green channels (left), as well as merged images (middle) are shown for easier comparison. Arrows indicate IS. E, The A/D PI and IS/D PI were calculated by dividing the average fluorescence intensity along stretches of distal axon or IS by the average fluorescence intensity along stretches of dendrites. Dendrites were identified by MAP2 immunoreactivity, and IS waS identified by ankG immunoreactivity. All transfected cells were scored regardless of expression levels. The average polarity indices for wild-type (WT) HA-NF (black bars; $n=29$ cells), HA-NF FIGQA (blue bars; $n=58$ cells), and CD4 $\Delta$ CT (yellow bars; $n=34$ cells) are plotted. Error bars indicate the SEM. ${ }^{*} p<0.001$, statistically significant difference from CD $4 \Delta$ CT by Mann-Whitney $U$ test ().

that compartmentalization of NF to the IS occurs by direct tethering attributable to ankyrin binding.

Diffusion behavior of NF in the axonal plasma membrane Using single-particle tracking, we show that NF is highly diffusible in young stage 3 neurons all along the axon but becomes restricted in its diffusion in the IS coincident with the accumulation of ankG there. This diffusion behavior is similar to that of several other proteins and even lipids in the IS (Winckler et al., 1999; Nakada et al., 2003). The time course of IS-restricted diffusion closely parallels the time course of ankG accumulation in the
IS. In our hands, a significant difference between IS and distal axon diffusion coefficients is observed clearly by DIV6. Nakada et al. (2003) observed a slowdown of lipid diffusion in the IS, but the observed IS restriction of diffusion was not fully established until DIV9/DIV10. This comparably delayed restriction of diffusion in their study might be attributable to different conditions of culturing neurons. It is also possible that lipids show a slower time course of acquiring restricted diffusion in the IS compared with NF. Future work directly comparing the two tracers in the same cultures will clarify this issue. Second, we observed that NF in ankG-positive ISs underwent confined rather than free diffusion, whereas NF on the distal axon of the same cells diffused freely. We achieved the high spatial and temporal resolution necessary to observe confinement by using beads. Single-fluorophore imaging might not give long enough trajectories and high enough signalto-noise ratios to clearly observe confinement. Confined diffusion might arise from diffusion through an area peppered with immobilized obstacles and/or from "corralling" by the cytoskeleton (Fujiwara et al., 2002). An $\alpha$ value of $\sim 0.5$ might also stem from averaging different diffusional behaviors between immobile and free, when NF would frequently become tethered to the cytoskeleton, and break free again. It is possible that crosslinking of $\mathrm{NF}$ by the multivalent bead contributed to the observation of confined diffusion. Importantly, multiple complex extracellular interactions take place at the IS in vivo (Ango et al., 2004; Eshed et al., 2005; Bruckner et al., 2006; John et al., 2006). The behavior of ligated NF might therefore be more relevant to biological functions in vivo. Whether in confinement or dynamic binding, the ankG/ $\beta \mathrm{IV}$-spectrin network in the IS might be directly involved in confining the diffusion of NF and creating the IS diffusion barrier.

Role for ankyrin in secretory traffic of L1 family members? Researchers wondered early on whether binding to ankyrin might already occur in the secretory pathway. Work in the 1990s identified Golgi-associated ankyrins and spectrins (Beck and Nelson, 1998; De Matteis and Morrow, 1998), and ankyrin binds some ankyrin-binding membrane proteins early in the secretory pathway (Gomez and Morgans, 1993). Several lines of evidence raised the possibility that ankyrin might be important for secretory targeting of neuronal ankyrin-binding membrane proteins as well (Garrido et al., 2003; Kizhatil and Bennett, 2004; Sherman et al., 2005; Xu and Shrager, 2005). Although Fache et al. (2004) demonstrated that selective removal from the somatodendritic membrane coupled to specific retention at the IS can in principle lead to IS localization of receptors, the idea that ankyrin binding might direct NF to the IS and nodes of Ranvier from the secretory pathway is attractive given the long distances between somata and distal nodes. Whether or not direct secretory targeting acts in addition to retention and selective removal to concentrate IS components at the IS remains to be tested directly. Because L1/ NgCAM lacking the ankyrin-binding motif still targets overwhelmingly to the axon, a role for ankyrin in secretory NgCAM trafficking is unlikely.

\section{Neurofascin accumulation in the IS during axonogenesis}

During axonogenesis in culture, NF can be transiently found broadly distributed along growing axons in cells in which ankG is not detectable at the IS. As soon as ankG becomes detectably enriched at the IS, NF staining also becomes IS enriched. The successive restriction of NF to the IS suggests that, in the absence of ankG, NF is found more broadly along axons, and its restrictions to the IS is attributable to increased ankG expression at the 
IS. Interestingly, mutation of the ankyrin binding motif of NF, although compromising IS localization, still results in threefold to fourfold enrichment in the axon. We therefore hypothesize that NF is trafficked with several-fold bias toward the axon from the secretory pathway using ankyrin-independent pathways. Interestingly, NgCAM travels indirectly to the axonal plasma membrane via dendrite-toaxon transcytosis (Wisco et al., 2003; Chang et al., 2006). Whether or not NF uses similar signals and pathways as NgCAM remains to be determined. Additionally, a possible role for endocytic retrieval of mistargeted NF from the somatodendritic domain as found for other proteins (Fache et al., 2004; Leterrier et al., 2006; Xu et al., 2006) remains to be investigated.

After reaching the axonal plasma membrane, NF molecules at the IS bind tightly to ankG, which retains them in the IS membrane. Non-IS NF molecules, conversely, diffuse rapidly and might accumulate at the IS by diffusional trapping. Alternatively or additionally, NF localized in the distal axon plasma membrane could be removed by endocytosis. The idea that NF first travels to the axon generally from the secretory pathway is also suggested by the transient broad axonal localization of NF in very young stage 3 neurons (phase IS1). An early axonal targeting might account for the proposed roles of NF and NrCAM in axon outgrowth and pathfinding in the chick and mouse visual system (Sakurai et al., 2001; Pruss et al., 2004, 2006; Zelina et al., 2005; Williams et al., 2006). After axon outgrowth and synaptogenesis are complete, secretory IS targeting might develop as a mature mechanism to efficiently enrich IS components at the IS later on. In myelinated axons, glial partners for nodal NF have been identified [i.e., gliomedin (Eshed et al., 2005; Koticha et al., 2006)], and these glial trans-binding partners likely serve important roles as well for accumulating and retaining NF at the node.

\section{Specificity of ankyrin isoform binding}

Given the colocalization of NF with ankG, we postulated that NF has an isoformbinding preference for ankG inside cells. In vitro biochemistry showed that $\mathrm{NF}$ binds ankR with higher affinity than ankB

(Davis et al., 1993; Michaely and Bennett, 1995). Although ankyrin recruitment assays in HEK 293 cells are less quantitative than in vitro biochemistry, they potentially preserve relevant cellular contexts of the interaction (Zhang et al., 1998). NF shows strong recruitment of ankG in HEK 293 cells (Zhang et al., 1998; Nishimura et al., 2003; this work). We show here in controlled, direct comparisons that NF shows significantly better recruitneurons (bottom).

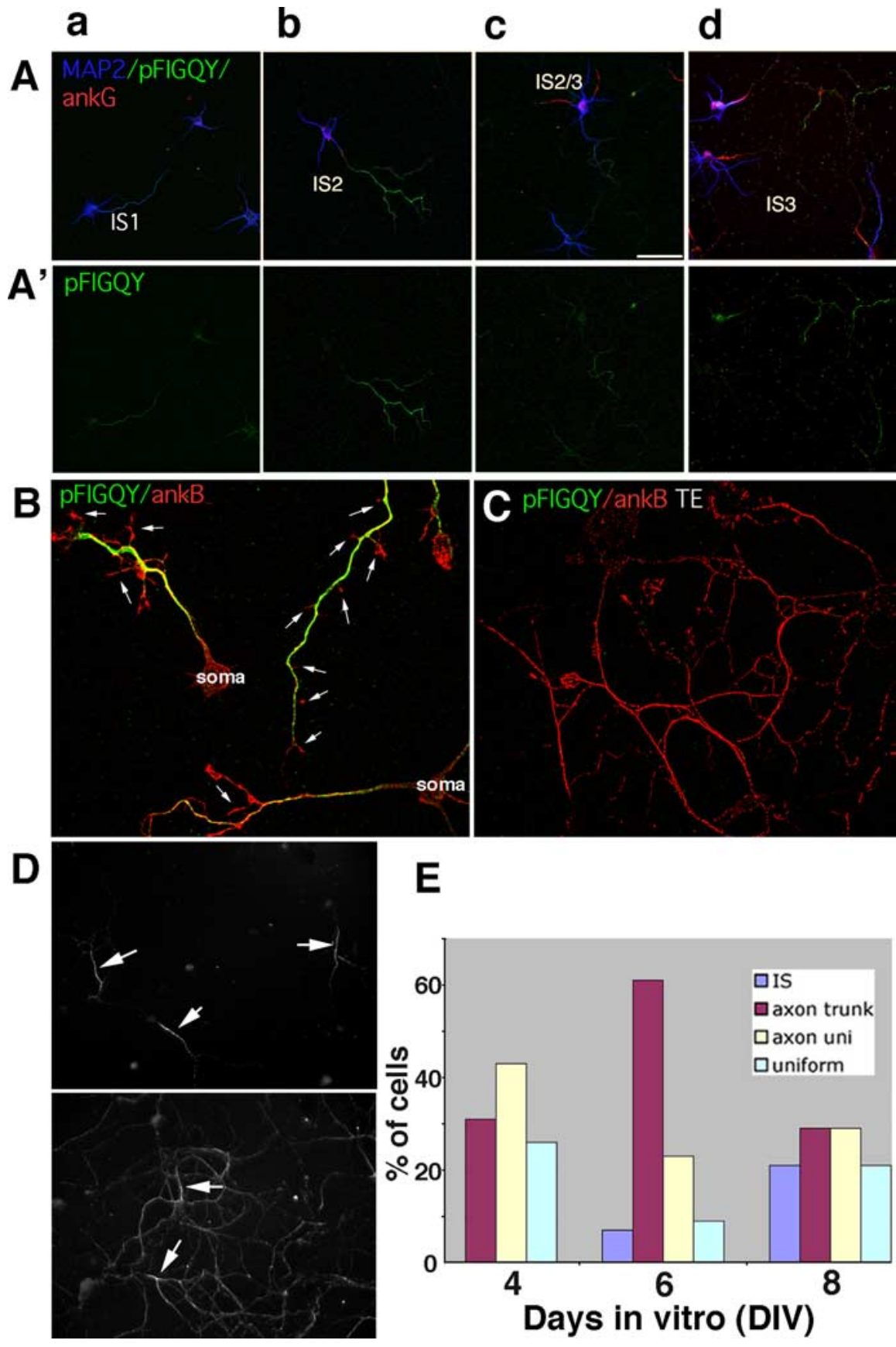

Figure 7. Temporal and spatial regulation of the phospho-FIGQY epitope during maturation of hippocampal neurons. $A, A^{\prime}$, phospho-FIGQY (green) staining patterns in hippocampal neurons at stages IS1 (a) , IS2 (b), late IS2 (c), and IS3 (d). Neurons were stained simultaneously with antibodies against ankG (red) and MAP2 (blue). $A^{\prime}$ shows green channels only. Scale bar, $50 \mu \mathrm{m}$. Quantification of the prevalence of different phospho-FIGQY staining patterns for a typical experiment is shown in $E . B, C$, Non-extracted $(\boldsymbol{B})$ or Triton X-100-extracted (TE; $\boldsymbol{C}$ ) neurons were stained against phospho-FIGQY (green) and ankB (red). Phospho-FIGQY staining (green) was essentially absent from filopodia and growth cones (arrows; $\boldsymbol{B}$ ) and was detergent soluble (C). D, DIV7 (top) and DIV11 (bottom) neurons were Triton X-100-extracted and stained against L1. L1 is preferentially detergent resistant at the IS in DIV7 (top) but becomes increasingly non-extractable along the whole axon in DIV11

ment of ankG than ankB over a range of expression levels, indicating that NF preferentially binds to ankG in cells.

The ankyrin-binding site on L1CAMs has been mapped for both NF and the Drosophila homolog neuroglian to the region surrounding the FIGQY motif (Hortsch et al., 1998; Zhang et al., 1998). FIGQA and FIGQD mutations in NF lead to virtual loss of ankG recruitment in HEK 293 cells (Zhang et al., 1998; this 
work). The FIGQF mutation, conversely, reportedly retained either full [neurofascin (Zhang et al., 1998)] or partial [neuroglian (Hortsch et al., 1998)] ankyrin-binding capacity. In our hands, NF FIGQF showed reduced ankG binding by several criteria: IS enrichment is drastically reduced compared with wild-type NF, HA-NF FGIQF is not retained in the IS after detergent extraction, and ankyrin-binding activity is reduced in HEK 293 recruitment assays (shown in supplemental Fig. 3, available at www.jneurosci. org as supplemental material). The isoform preference of NF for ankG provides a mechanism for tethering NF specifically in the IS but not along the axon.

\section{Ankyrin binding in L1 family member biology}

The role of ankyrin binding for the function of L1 and NF has been investigated in multiple studies. Ankyrin binding modulates L1-mediated neurite outgrowth (Gil et al., 2003; Nishimura et al., 2003; Whittard et al., 2006) and NF adhesion in culture assays (Tuvia et al., 1997). We show that NF binds strongly to ankG at the IS at all times of axonogenesis. It does not appear to bind significantly to ankB. This strong binding of NF and ankG might reflect a stabilizing role for NF and ankG in building the IS as a stable specialized membrane domain. ankG and ankB are also spatially segregated in myelinated axons and might contribute to organizing nodal and paranodal domains as well (Ogawa et al., 2006). L1, conversely, shows spatial and temporal regulation of its ankyrin binding. During early axon outgrowth, L1 is not stably associated with ankB along axons and shows higher levels of phosphorylation of the FIGQY motif. This observation supports the notion that axon outgrowth requires dynamic association of L1 with cytoskeletal elements, especially dynamic actin filaments (Gil et al., 2003; Whittard et al., 2006). Ankyrin binding stabilizes L1 in the membrane and reduces engagement of the dynamic actin flow (Gil et al., 2003) (see also Nishimura et al., 2003). Regulation of FIGQY phosphorylation therefore can modulate the extent to which L1 engages either F-actin or ankyrin. Growth factors as well as ligand binding of L1 are potentially important regulators of ankyrin binding (Cheng et al., 2005; Whittard et al., 2006). It is surprising that our results show low/nondetectable levels of phospho-FIGQY staining in growth cones and filopodia in which most of the dynamic actin resides. After axon outgrowth is mostly complete, $\mathrm{L} 1$ shows reduced phosphorylation of FIGQY and is more tightly associated with insoluble cytoskeleton along axons. L1-ankB interactions might now stabilize the membrane. Consistent with this notion, optic nerve axons grow out normally and contain L1 on their surfaces in ankB knock-out mice, but the optic nerve axons degenerate later on (Scotland et al., 1998). Our findings in culture mirror data from developing cortex in which L1 initially codistributes with ERM proteins (ezrin, radixin, moesin proteins) but later shows increased colocalization with ankB (Mintz et al., 2003). Similar to our neuronal cultures, phosphoFIGQY content of L1 family members also decreases during development in vivo (Jenkins et al., 2001). More work is needed to understand what mechanisms other than phosphorylation potentially modulate ankyrin binding of L1.

\section{Conclusion}

In this work, we asked how two different L1 family members achieve their steady-state distribution during axon outgrowth. $\mathrm{NF}$ is an ankyrin-binding adhesion molecule localized to the IS and nodes of Ranvier. Both of these locales also accumulate ankG. $\mathrm{L} 1 / \mathrm{NgCAM}$ codistributes along axons with ankB rather than with ankG. We show that $\mathrm{L} 1$ and ankB are axonally enriched starting in early stage 3 neurons, mirroring their mature steady-state dis- tribution. The IS-resident protein NF, conversely, is transiently more broadly distributed along axons and, coordinately with ankG, accumulates to higher and higher levels in the IS while gradually disappearing from the more distal portions of the axon. Both L1/NgCAM and NF show slow diffusion and low detergent extractability specifically in the IS. We hypothesize that NF is initially targeted in an ankyrin-independent manner to the axon generally and accumulates at the IS in a subsequent step after reaching the axonal plasma membrane attributable to its higher binding to ankG. Multiple signals might also be used by nodal potassium channels for IS accumulation (Chung et al., 2006). For L1, we propose that it also is targeted to axons by an ankyrinindependent mechanism and that its ankyrin-binding capacity is temporally and spatially regulated by phosphorylation. The ability to regulate the ankyrin-binding capacity of L1 is likely important for controlling the dynamic functions of L1 in different places and at different times (Garver et al., 1997; Tuvia et al., 1997; Jenkins et al., 2001; Kizhatil et al., 2002; Godenschwege et al., 2006; Whittard et al., 2006). More work is needed to fully dissect when and where L1 binds ankyrin and to what effect.

\section{References}

Ango F, di Cristo G, Higashiyama H, Bennett V, Wu P, Huang ZJ (2004) Ankyrin-based subcellular gradient of neurofascin, an immunoglobulin family protein, directs GABAergic innervation at purkinje axon initial segment. Cell 119:257-272.

Beck KA, Nelson WJ (1998) A spectrin membrane skeleton of the Golgi complex. Biochem Biophys Acta 14:153-160.

Bennett V, Chen L (2001) Ankyrins and cellular targeting of diverse membrane proteins to physiological sites. Curr Opin Cell Biol 13:61-67.

Bennett V, Lambert S (1999) Physiological roles of axonal ankyrins in survival of premyelinated axons and localization of voltage-gated sodium channels. J Neurocytol 28:303-318.

Berghs S, Aggujaro D, Dirkx RJ, Maksimova E, Stabach P, Hermel JM, Zhang JP, Philbrick W, Slepnev V, Ort T, Solimena M (2000) betaIV spectrin, a new spectrin localized at axon initial segments and nodes of ranvier in the central and peripheral nervous system. J Cell Biol 151:985-1002.

Boiko T, Van Wart A, Caldwell JH, Levinson SR, Trimmer JS, Matthews G (2003) Functional specialization of the axon initial segment by isoformspecific sodium channel targeting. J Neurosci 23:2306-2313.

Bruckner G, Szeoke S, Pavlica S, Grosche J, Kacza J (2006) Axon initial segment ensheathed by extracellular matrix in perineuronal nets. Neuroscience 138:365-375.

Chang MC, Wisco D, Ewers H, Norden C, Winckler B (2006) Inhibition of sphingolipid synthesis affects kinetics but not fidelity of L1/NgCAM transport along direct but not transcytotic axonal pathways. Mol Cell Neurosci 31:525-538.

Cheng L, Lemmon S, Lemmon V (2005) RanBPM is an L1-interacting protein that regulates L1-mediated mitogen-activated protein kinase activation. J Neurochem 94:1102-1110.

Chung HJ, Jan YN, Jan LY (2006) Polarized axonal surface expression of neuronal KCNQ channels is mediated by multiple signals in the KCNQ2 and KCNQ3 C-terminal domains. Proc Natl Acad Sci USA 103:8870-8875.

Coombs JS, Curtis DR, Eccles JC (1957) The generation of impulses in motoneurones. J Physiol (Lond) 139:232-249.

Custer AW, Kazarinova-Noyes K, Sakurai T, Xu X, Simon W, Grumet M, P. S (2003) The role of the ankyrin-binding protein NrCAM in node of Ranvier formation. J Neurosci 23:10032-10039.

Davis JQ, McLaughlin T, Bennett V (1993) Ankyrin-binding proteins related to nervous system cell adhesion molecules: candidates to provide transmembrane and intercellular connections in adult brain. J Cell Biol 121:121-133.

Davis JQ, Lambert S, Bennett V (1996) Molecular composition of the node of Ranvier: identification of ankyrin-binding cell adhesion molecules neurofascin (mucin+/third FNIII-) and NrCAM at nodal axonal segments. J Cell Biol 135:1355-1367.

De Matteis MA, Morrow JS (1998) The role of ankyrin and spectrin in membrane transport and domain formation. Curr Opin Cell Biol 10:542-549. 
Dickson TL, Mintz CD, Benson DL, Salton SRJ (2002) Functional binding interaction identified between the axonal CAM L1 and members of the ERM family. J Cell Biol 157:1105-1112.

Dotti CG, Sullivan CA, Banker GA (1988) The establishment of polarity by hippocampal neurons in culture. J Neurosci 8:1454-1468.

Eshed Y, Feinberg K, Poliak S, Sabanay H, Sarig-Nadir O, Spiegel I, Bermingham JRJ, Peles E (2005) Gliomedin mediates Schwann cell-axon interaction and the molecular assembly of the nodes of Ranvier. Neuron 47:215-229.

Ewers H, Smith AE, Sbalzarini IF, Lilie H, Koumoutsakos P, Helenius A (2005) Single-particle tracking of murine polyoma virus-like particles on live cells and artificial membranes. Proc Natl Acad Sci USA 102:15110-15115.

Fache MP, Moussif A, Fernandes F, Giraud P, Garrido JJ, Dargent B (2004) Endocytotic elimination and domain-selective tethering constitute a potential mechanism of protein segregation at the axonal initial segment. J Cell Biol 166:571-578.

Feder TJ, Brust-Mascher I, Slattery JP, Baird B, Webb WW (1996) Constrained diffusion or immobile fraction on cell surfaces: a new interpretation. Biophys J 70:2767-2773.

Frankenhaeuser B, Huxley AF (1964) The action potential in the myelinated nerve fiber of Xenopus laevis as computed on the basis of voltage clamp data. J Physiol (Lond) 171:302-315.

Fransen E, Lemmon V, Van Camp G, Vits L, Coucke P, Willems PJ (1995) CRASH syndrome: clinical spectrum of corpus callosum hypoplasia, retardation, adducted thumbs, spastic paraparesis and hydrocephalus due to mutations in one single gene, L1. Eur J Hum Genet 3:273-284.

Fujiwara T, Ritchie K, Murakoshi H, Jacobson K, Kusumi A (2002) Phospholipids undergo hop diffusion in compartmentalized cell membrane. J Cell Biol 157:1071-1081.

Garrido JJ, Fernandes F, Giraud P, Mouret I, Pasqualini E, Fache MP, Jullien F, Dargent B (2001) Identification of an axonal determinant in the C-terminus of the sodium channel $\mathrm{Na}(\mathrm{v})$ 1.2. EMBO J 20:5950-5961.

Garrido JJ, Giraud P, Carlier E, Fernandes F, Moussif A, Fache MP, Debanne D, Dargent B (2003) A targeting motif involved in sodium channel clustering at the axonal initial segment. Science 300:2091-2094.

Garver TD, Ren Q, Tuvia S, Bennett V (1997) Tyrosine phosphorylation at a site highly conserved in the L1 family of cell adhesion molecules abolishes ankyrin binding and increases lateral mobility of neurofascin. J Cell Biol 137:703-714.

Gil OD, Sakurai T, Bradley AE, Fink MY, Cassella MR, Kuo JA, Felsenfeld DP (2003) Ankyrin binding mediates L1CAM interactions with static components of the cytoskeleton and inhibits retrograde movement of L1CAM on the cell surface. J Cell Biol 162:719-730.

Godenschwege TA, Kristiansen LV, Uthaman SB, Hortsch M, Murphey RK (2006) A conserved role for Drosophila Neuroglian and human L1-CAM in central-synapse formation. Curr Biol 16:12-23.

Gomez S, Morgans C (1993) Interaction between band 3 and ankyrin begins in early compartments of the secretory pathway and is essential for band 3 processing. J Biol Chem 268:19593-19603.

Haspel J, Grumet M (2003) The L1CAM extracellular region: a multidomain protein with modular and cooperative binding modes. Front Biosci 8:s1210-s1225.

Hedstrom KL, Rasband MN (2006) Intrinsic and extrinsic determinants of ion channel localization in neurons. J Neurochem 98:1345-1352.

Hortsch M (2000) Structural and functional evolution of the L1 family: are four adhesion molecules better than one? Mol Cell Neurosci 15:1-10.

Hortsch M, Homer D, Malhotra JD, Chang S, Frankel J, Jefford G, Dubreuil RR (1998) Structural requirements for outside-in and inside-out signaling by Drosophila neuroglian, a member of the L1 family of cell adhesion molecules. J Cell Biol 142:251-261.

Jenkins SM, Bennett V (2001) Ankyrin-G coordinates assembly of the spectrin-based membrane skeleton, voltage-gated sodium channels, and L1 CAMs at Purkinje neuron initial segments. J Cell Biol 155:739-746.

Jenkins SM, Kizhatil K, Kramarcy NR, Sen A, Sealock R, Bennett V (2001) FIGQY phosphorylation defines discrete populations of L1 cell adhesion molecules at sites of cell-cell contact and in migrating neurons. J Cell Sci 114:3823-3835.

John N, Krugel H, Frischknecht R, Smalla KH, Schultz C, Kreutz MR, Gundelfinger ED, Seidenbeche RC (2006) Brevican-containing perineuronal nets of extracellular matrix in dissociated hippocampal primary cultures. Mol Cell Neurosci 31:774-784.
Johnson EM, Kinoshita Y, Weinreb DB, Wortman MJ, Simon R, Khalili K, Winckler B, Gordon J (2006) Role of Pur alpha in targeting mRNA to sites of translation in hippocampal neuronal dendrites. J Neurosci Res 83:929-943.

Kamiguchi H (2003) The mechanism of axon growth: what we have learned from the cell adhesion molecule L1. Mol Neurobiol 28:219-228.

Kamiguchi H, Long KE, Pendergast M, Schaefer AW, Rapoport I, Kirchhausen T, Lemmon V (1998) The neural cell adhesion molecule L1 interacts with the AP-2 adaptor and is endocytosed via the clathrinmediated pathway. J Neurosci 18:5311-5321.

Khaliq ZM, Raman IM (2006) Relative contributions of axonal and somatic $\mathrm{Na}$ channels to action potential initiation in cerebellar Purkinje neurons. J Neurosci 26:1935-1944.

Kizhatil K, Bennett V (2004) Lateral membrane biogenesis in human bronchial epithelial cells requires $190-\mathrm{kDa}$ ankyrin-G. J Biol Chem 279:16706-16714.

Kizhatil K, Wu YX, Sen A, Bennett V (2002) A new activity of doublecortin in recognition of the phospho-FIGQY tyrosine in the cytoplasmic domain of neurofascin. J Neurosci 22:7948-7958.

Kordeli E, Lambert S, Bennett V (1995) AnkyrinG. A new ankyrin gene with neural specific isoforms localized at the axon initial segment and node of Ranvier. J Biol Chem 270:2352-2359.

Koticha D, Maurel P, Zanazzi G, Kane-Goldsmith N, Basak S, Babiarz J, Salzer J, Grumet M (2006) Neurofascin interactions play a critical role in clustering sodium channels, ankyrin G and beta IV spectrin at peripheral nodes of Ranvier. Dev Biol 293:1-12.

Kusumi A, Sako Y, Yamamoto M (1993) Confined lateral diffusion of membrane receptors as studied by single particle tracking (nanovid microscopy). Effects of calcium induced differentiation in cultured epithelial cells. Biophys J 65:2021-2040.

Kusumi A, Sako Y, Fujiwara T, Tomishige M (1998) Application of laser tweezers to studies of the fences and tethers of the membrane skeleton that regulate the movements of plasma membrane proteins. Methods Cell Biol 55:173-194.

Lemaillet G, Walker B, Lambert S (2003) Identification of a conserved ankyrin-binding motif in the family of sodium channel alpha subunits. J Biol Chem 278:27333-27339.

Leterrier C, Laine J, Darmon M, Boudin H, Rossier J, Lenkei Z (2006) Constitutive activation drives compartment-selective endocytosis and axonal targeting of type 1 cannabinoid receptors. J Neurosci 26:3141-3153.

Michaely P, Bennett V (1995) Mechanism for binding site diversity on ankyrin. Comparison of binding sites on ankyrin for neurofascin and the Cl-/HCO3- anion exchanger. J Biol Chem 270:31298-31302.

Mintz CD, Dickson TC, Gripp ML, Salton SR, Benson DL (2003) ERMs colocalize transiently with L1 during neocortical axon outgrowth. J Comp Neurol 464:438-448.

Mohler PJ, Gramolini AO, Bennett V (2002) The ankyrin-B C-terminal domain determines activity of ankyrin-B/G chimeras in rescue of abnormal inositol 1,4,5-trisphosphate and ryanodine receptor distribution in ankyrin-B (-/-) neonatal cardiomyocytes. J Biol Chem 277:10599-10607.

Mohler PJ, Davis JQ, Bennett V (2005) Ankyrin-B coordinates the Na/K ATPase, $\mathrm{Na} / \mathrm{Ca}$ exchanger, and InsP3 receptor in a cardiac T-tubule/SR microdomain. PLoS Biol 3:e423.

Nakada C, Ritchie K, Oba Y, Nakamura M, Hotta Y, Iino R, Kasai RS, Yamaguchi K, Fujiwara T, Kusumi A (2003) Accumulation of anchored proteins forms membrane diffusion barriers during neuronal polarization. Nat Cell Biol 5:626-632.

Nishimura K, Yoshihara F, Tojima T, Ooashi N, Yoon W, Mikoshiba K, Bennett V, Kamiguchi H (2003) L1-dependent neuritogenesis involves ankyrinB that mediates L1-CAM coupling with retrograde actin flow. J Cell Biol 163:1077-1088.

Ogawa Y, Schafer DP, Horresh I, Bar V, Hales K, Yang Y, Susuki K, Peles E, Stankewich MC, Rasband MN (2006) Spectrins and ankyrinB constitute a specialized paranodal cytoskeleton. J Neurosci 26:5230-5239.

Palmer LM, Stuart GJ (2006) Site of action potential initiation in layer 5 pyramidal neurons. J Neurosci:1854-1863.

Pan Z, Kao T, Horvath Z, Lemos J, Sul JY, Cranstoun SD, Bennett V, Scherer SS, Cooper EC (2006) A common ankyrin-G-based mechanism retains KCNQ and NaV channels at electrically active domains of the axon. J Neurosci 26:2599-2613.

Pruss T, Niere M, Kranz EU, Volkmer H (2004) Homophilic interactions of 
chick neurofascin in trans are important for neurite induction. Eur J Neurosci 20:3184-3188.

Pruss T, Kranz EU, Niere M, Volkmer H (2006) A regulated switch of chick neurofascin isoforms modulates ligand recognition and neurite extension. Mol Cell Neurosci 31:354-365.

Sakurai T, Lustig M, Babiarz J, Furley AJ, Tait S, Brophy PJ, Brown SA, Brown LY, Mason CA, Grumet M (2001) Overlapping functions of the cell adhesion molecules $\mathrm{Nr}$-CAM and L1 in cerebellar granule cell development. J Cell Biol 154:1259-1273.

Salzer JL (2003) Polarized domains of myelinated axons. Neuron 40:297-318.

Scotland P, Zhou D, Benveniste H, Bennett V (1998) Nervous system defects of AnkyrinB (-/ ) mice suggest functional overlap between the cell adhesion molecule L1 and 440-kD AnkyrinB in premyelinated axons. J Cell Biol 143:1305-1315.

Sherman DL, Tait S, Melrose S, Johnson R, Zonta B, Court FA, Macklin WB, Meek S, Smith AJ, Cottrell DF, Brophy PJ (2005) Neurofascins are required to establish axonal domains for saltatory conduction. Neuron 48:737-742.

Tuvia S, Garver TD, Bennett V (1997) The phosphorylation state of the FIGQY tyrosine of neurofascin determines ankyrin-binding activity and patterns of cell segregation. Proc Natl Acad Sci USA 94:12957-12962.

Tuvia S, Buhusi M, Davis L, Reedy M, Bennett V (1999) Ankyrin-B is required for intracellular sorting of structurally diverse $\mathrm{Ca}^{2+}$ homeostasis proteins. J Cell Biol 147:995-1008.

Van Wart A, Matthews G (2006) Impaired firing and cell-specific compensation in neurons lacking navl.6 sodium channels. J Neurosci 26:7172-7180.

Volkmer H, Leuschner R, Zacharias U, Rathjen FG (1996) Neurofascin induces neurites by heterophilic interactions with axonal NrCAM while NrCAM requires F11 on the axonal surface to extend neurites. J Cell Biol 135:1059-1069.
Whittard JD, Sakurai T, Cassella MR, Gazdoiu M, Felsenfeld DP (2006) MAP kinase pathway-dependent phosphorylation of the L1-CAM ankyrin binding site regulates neuronal growth. Mol Biol Cell 17:2696-2706.

Williams SE, Grumet M, Colman DR, Henkemeyer M, Mason CA, Sakurai T (2006) A role for Nr-CAM in the patterning of binocular visual pathways. Neuron 50:535-547.

Winckler B, Forscher P, Mellman I (1999) A diffusion barrier maintains distribution of membrane proteins in polarized neurons. Nature 397:698-701.

Wisco D, Anderson ED, Chang MC, Norden C, Boiko T, Folsch H, Winckler B (2003) Uncovering multiple axonal targeting pathways in hippocampal neurons. J Cell Biol 162:1317-1328.

Xu J, Zhu Y, Heinemann SF (2006) Identification of sequence motifs that target neuronal nicotinic receptors to dendrites and axons. J Neurosci 26:9780-9793.

Xu X, Shrager P (2005) Dependence of axon initial segment formation on $\mathrm{Na}^{+}$channel expression. J Neurosci Res 79:428-441.

Zelina P, Avci HX, Thelen K, Pollerberg GE (2005) The cell adhesion molecule NrCAM is crucial for growth cone behaviour and pathfinding of retinal ganglion cell axons. Development 132:3609-3618.

Zhang X, Bennett V (1998) Restriction of 480/270-kD ankyrin G to axon proximal segments requires multiple ankyrin G-specific domains. J Cell Biol 142:1571-1581.

Zhang X, Davis JQ, Carpenter S, Bennett V (1998) Structural requirements for association of neurofascin with ankyrin. J Biol Chem 273: 30785-30794.

Zhou D, Lambert S, Malen PL, Carpenter S, Boland LM, Bennett V (1998) AnkyrinG is required for clustering of voltage-gated $\mathrm{Na}$ channels at axon initial segments and for normal action potential firing. J Cell Biol 143: 1295-1304. 